\title{
A compendium of comparison function results
}

\author{
Christopher M. Kellett
}

Received: 28 November 2012 / Accepted: 25 February 2014 / Published online: 20 March 2014 (C) The Author(s) 2014. This article is published with open access at Springerlink.com

\begin{abstract}
The use of comparison functions has become standard in systems and control theory, particularly for the purposes of studying stability properties. The use of these functions typically allows elegant and succinct statements of stability properties such as asymptotic stability and input-to-state stability and its several variants. Furthermore, over the last 20 years several inequalities involving these comparison functions have been developed that simplify their manipulation in the service of proving more significant results. Many of these inequalities have appeared in the body of proofs or in appendices of various papers. Our goal herein is to collect these inequalities in one place.
\end{abstract}

Keywords Comparison functions $\cdot$ Stability theory $\cdot$ Nonlinear systems

\section{History}

Jose Massera appears to have been the first scholar to introduce comparison functions to the study of stability theory in 1956 [26]. In particular, to elegantly capture the notion of (local) positive definiteness he relied on a function $a: \mathbb{R}_{\geq 0} \rightarrow \mathbb{R}_{\geq 0}$ with

" $a(r)$ being continuous and increasing when $r>0, a(0)=0$."

To further describe a function having an infinitely small upper bound, he then used a function $b: \mathbb{R}_{\geq 0} \rightarrow \mathbb{R}_{\geq 0}$ "having the same properties of $a(r)$ ". 1

\footnotetext{
${ }^{1}$ It is worth remarking that in his 1949 manuscript studying similar stability problems, Massera [25] does not use comparison functions, but instead uses the more classical $\varepsilon-\delta$ formulations, indicating their development sometime between 1949 [25] and 1956 [26].
}

C. M. Kellett ( $\bowtie)$

School of Electrical Engineering and Computer Science, University of Newcastle,

Callaghan, NSW 2308, Australia

e-mail: Chris.Kellett@ newcastle.edu.au 
In his 1959 manuscript, Wolfgang Hahn [11] termed such functions as class- $\mathcal{K}$ functions ${ }^{2}$ and also introduced the terminology "decrescent" for what Lyapunov had originally termed "an infinitely small upper limit" [24]. However, in what remains a remarkably modern text, it was in his 1967 manuscript [12] where Hahn not only introduced the additional function classes of class- $\mathcal{L}$ and class- $\mathcal{K} \mathcal{L}$, but also made significant use of these comparison functions and their properties.

Following Hahn's text, functions of class- $\mathcal{K}$ appeared occasionally in works through the 1970s and 1980s. However, the use of these functions was largely limited to the characterization of positive definite and decrescent functions and the properties that Hahn elucidated in [12] were essentially ignored [21,32,34,41,42].

Sontag's seminal 1989 paper [35] introducing the notion of input-to-state stability represented a return to the elegant formulations first presented in [12]. In particular, Sontag took as the definition of global asymptotic stability the formulation involving a class- $\mathcal{K} \mathcal{L}$ function first proposed in [12, Equation 26.2]. To study systems under the influence of inputs, Sontag then augmented the class- $\mathcal{K} \mathcal{L}$ formulation of global asymptotic stability with a class- $\mathcal{K}$ function of the input.

Since the appearance of [35], the use of comparison functions in the analysis of stability and robustness for nonlinear systems has become standard. Many useful inequalities and other relationships have been developed for comparison functions. Unfortunately, these results tend to be reported in appendices or within the context of proving some larger results relating to stability or robustness. Our goal here is a modest one: to collect some of the most useful comparison function inequalities and relationships in one place.

The manuscript is organized as follows: in Sect. 2 we provide the standard comparison function definitions and review some well-known facts. In Sect. 3, we provide lemmas that give upper bounds on a given comparison function. By contrast, in Sect. 4, we provide lemmas that give lower bounds on a given function. Section 5 provides lemmas on differential inequalities involving comparison functions. In an overlap of terminology that should cause no confusion, these lemmas are frequently referred to as comparison lemmas. In Sect. 6, we provide some other useful relationships involving comparison functions. In Sect. 7, we briefly mention a few recently defined function classes that we believe are likely to be more widely used in the future. Finally, in Sect. 8 we discuss a generalization of class- $\mathcal{K}$ functions, termed monotone aggregation functions, that are defined on $\mathbb{R}_{\geq 0}^{n}$ rather than $\mathbb{R}_{\geq 0}$. For the purpose of illustrating some of the proof techniques, proofs of selected results are included in the Appendix.

\section{Definitions and obvious facts}

In what follows, we denote integers by $\mathbb{Z}$ and real numbers by $\mathbb{R}$. Restrictions to subsets of $\mathbb{Z}$ or $\mathbb{R}$ will be denoted by subscripts such as $\mathbb{R}_{\geq 0} \subset \mathbb{R}$ for the nonnegative real half-line or by the standard notation $[a, b) \subset \mathbb{R}$ for the half-open interval. We denote

2 It is known that Hahn was aware of Massera's work [26] as Massera submitted an Erratum for [26] to Annals of Mathematics on 13 January 1958 where he cites Hahn as having brought an error in the original paper to his attention. 
$n$-dimensional Euclidean space by $\mathbb{R}^{n}$ and use $|\cdot|$ to denote the norm. We will use both parenthesis and the symbol $\circ$ to denote function composition; i.e., for functions $\alpha_{1}, \alpha_{2}: \mathbb{R}_{\geq 0} \rightarrow \mathbb{R}_{\geq 0}$, we will use either $\alpha_{1}\left(\alpha_{2}(s)\right)$ or $\alpha_{1} \circ \alpha_{2}(s)$ for all $s \in \mathbb{R}_{\geq 0}$, where the choice is always made to improve readability.

As previously mentioned, $\mathrm{Hahn}^{3}$ first defined functions of class- $\mathcal{K}$ in [11], though such functions had been previously used in stability analysis by Massera in [26]. In [12, Defn 2.5] this definition is immediately used to define the notion of a stable equilibrium point rather than as a precursor to using such functions to characterize positive definiteness (as done in [11,26,32,41]).

Definition 1 A function $\alpha: \mathbb{R}_{\geq 0} \rightarrow \mathbb{R}_{\geq 0}$ is said to be of class- $\mathcal{K}(\alpha \in \mathcal{K})$ if it is continuous, zero at zero, and strictly increasing. For some $a \in \mathbb{R}_{>0}$, a function $\alpha:[0, a) \rightarrow \mathbb{R}_{\geq 0}$ is said to be of class- $\mathcal{K}_{[0, a)}$, if it is continuous, zero at zero, and strictly increasing.

As cited in the introduction, the first use of class- $\mathcal{K}$ functions was to characterize the concept of a locally positive definite function. To see that this is indeed the case, we follow the same argument as [12]. A positive definite function is understood as a function that is zero at the origin, and (strictly) positive at every other point. Given, then, a continuous locally positive definite function $\rho: \mathbb{R}^{n} \rightarrow \mathbb{R}_{\geq 0}$ on a closed ball of radius $c$ (denoted by $\overline{\mathcal{B}}_{c} \subset \mathbb{R}^{n}$ ), we can define a continuous nondecreasing function $\hat{\alpha}: \mathbb{R}_{\geq 0} \rightarrow \mathbb{R}_{\geq 0}$ by

$$
\hat{\alpha}(s) \doteq \min _{\left\{x \in \overline{\mathcal{B}}_{c}:|x| \geq s\right\}} \rho(x), \quad \forall s \in[0, c] .
$$

Since $\hat{\alpha}(s)$ is strictly positive for $(0, c]$, it is possible to lower bound this nondecreasing function by one that is strictly increasing to obtain the desired result. Note that globally positive definite continuous functions that satisfy $\lim _{s \rightarrow \infty} \rho(s)=0$ cannot be bounded from below by a class- $\mathcal{K}$ function. A comparison function lower bound for globally positive definite continuous functions is presented in Lemma 18.

Hahn [11] introduced the term radially unbounded and in [12] characterized a radially unbounded function as one that is lower bounded by a class- $\mathcal{K}$ function that approaches infinity as its argument approaches infinity. Hahn cites [4] for introducing the same notion under the terminology " $\alpha$ becomes infinitely large". Sontag [35] introduced the now standard notation $\mathcal{K}_{\infty}$ for such functions. ${ }^{4}$

Definition 2 A function $\alpha: \mathbb{R}_{\geq 0} \rightarrow \mathbb{R}_{\geq 0}$ is said to be of class- $\mathcal{K}_{\infty}\left(\alpha \in \mathcal{K}_{\infty}\right)$ if $\alpha \in \mathcal{K}$ and, in addition, $\lim _{s \rightarrow \infty} \alpha(s)=\infty$.

\footnotetext{
3 It has been speculated that Hahn's usage of the letter $\mathcal{K}$ was in reference to Kamke, though Hahn himself never apparently stated this. Functions similar to class- $\mathcal{K}$ functions do appear in [28] and are, in fact, denoted by $K(\cdot)$. In a similar vein, [27] makes use of a bounding function that is continuous and monotonically decreasing to zero on $(0, \infty)$.

4 Observe that class- $\mathcal{K}_{[0, \infty)}$ and class- $\mathcal{K}_{\infty}$ are not the same. In fact, class- $\mathcal{K}_{[0, \infty)}$ is the same as class- $\mathcal{K}$ since the subscript interval refers to the domain of definition of a function. By contrast, the subscript of $\mathcal{K}_{\infty}$ refers to the behavior of the function in the limit as its argument goes to infinity.
} 
One of the most useful properties of class- $\mathcal{K}$ and class- $\mathcal{K}_{\infty}$ functions is their invertibility. First consider $\alpha \in \mathcal{K} \backslash \mathcal{K}_{\infty}$; i.e., $\alpha \in \mathcal{K}$ but $\alpha \notin \mathcal{K}_{\infty}$. Since $\alpha \in \mathcal{K} \backslash \mathcal{K}_{\infty}$ is continuous and strictly increasing there exists a constant $a \in \mathbb{R}_{>0}$ such that $\lim _{s \rightarrow \infty} \alpha(s)=a$, the inverse function exists on $[0, a)$, and the inverse function is also continuous and strictly increasing; i.e., the function $\alpha^{-1}:[0, a) \rightarrow \mathbb{R}_{\geq 0}$ is continuous, zero at zero, strictly increasing, and hence of class- $\mathcal{K}_{[0, a)}$. The inverse function also satisfies $\lim _{s \rightarrow a} \alpha^{-1}(s)=\infty$. In the case where $\alpha \in \mathcal{K}_{\infty}$, we observe that the inverse function will be defined globally and consequently $\alpha^{-1} \in \mathcal{K}_{\infty}$. We thus observe that class- $\mathcal{K}_{\infty}$ functions are the set of homeomorphisms on the nonnegative real half-line, $\mathbb{R}_{\geq 0}$.

Hahn [12] observed that if $\alpha_{1}, \alpha_{2} \in \mathcal{K}_{\infty}$ and if $\alpha_{1}(s) \leq \alpha_{2}(s)$ for all $s \in \mathbb{R}_{\geq 0}$, then $\alpha_{1}^{-1}(s) \geq \alpha_{2}^{-1}(s)$. This is straightforward to see by considering $r=\alpha_{1}(s)$ in the first inequality. This property also holds for two class- $\mathcal{K}$ functions, though only on $[0, a)$ where $a=\lim _{s \rightarrow \infty} \min \left\{\alpha_{1}(s), \alpha_{2}(s)\right\}$.

Three further useful and straightforward properties are related to the sum, maximum, or minimum of class- $\mathcal{K}$ functions. Suppose we have a finite number of functions $\alpha_{i} \in \mathcal{K}$ where $i \in\{1, \ldots, N\}$ for some finite $N \in \mathbb{Z}_{>0}$. Then, the function $\alpha: \mathbb{R}_{\geq 0} \rightarrow \mathbb{R}_{\geq 0}$ defined by any of

$$
\begin{aligned}
& \alpha(s) \doteq \sum_{i=1}^{N} \alpha_{i}(s) \\
& \alpha(s) \doteq \max _{i \in\{1, \ldots, N\}} \alpha_{i}(s), \quad \text { or } \\
& \alpha(s) \doteq \min _{i \in\{1, \ldots, N\}} \alpha_{i}(s),
\end{aligned}
$$

for all $s \in \mathbb{R}_{\geq 0}$ is of class- $\mathcal{K}$. Furthermore, for the summation or maximum, if $\alpha_{i} \in \mathcal{K}_{\infty}$ for any $i \in\{1, \ldots, N\}$, then $\alpha \in \mathcal{K}_{\infty}$. Note that maximization and summation are specific instances of the monotone aggregation functions discussed in Sect. 8.

Hahn introduced class- $\mathcal{L}$ functions in [12, Defn 2.6] as a precursor to defining attractivity of an equilibrium point.

Definition 3 A function $\sigma: \mathbb{R}_{\geq 0} \rightarrow \mathbb{R}_{>0}$ is said to be of class- $\mathcal{L}(\sigma \in \mathcal{L})$, if it is continuous, strictly decreasing, ${ }^{5}$ and $\lim _{s \rightarrow \infty} \sigma(s)=0$.

Note that, similar to class- $\mathcal{K}$ functions, functions of class- $\mathcal{L}$ are invertible on their range and the inverse is itself (nearly) class- $\mathcal{L}$ on the range of the original function; i.e., for $\sigma \in \mathcal{L}$, the inverse $\sigma^{-1}:(0, \sigma(0)] \rightarrow \mathbb{R}_{\geq 0}$ is continuous, strictly decreasing, and $\lim _{s \rightarrow 0} \sigma^{-1}(s)=+\infty$.

\footnotetext{
5 Note that some authors only assume that functions of class- $\mathcal{L}$ are nonincreasing. In many cases, this is sufficient. However, we prefer the symmetry with the strictly increasing property of class- $\mathcal{K}$ functions and the clean results on class- $\mathcal{L}$ functions that the assumption of strictly decreasing enables. Note that, since functions of class- $\mathcal{L}$ are strictly decreasing and go to zero in the limit, these functions are also strictly positive. This is explicit in the definition in that the range of class- $\mathcal{L}$ functions is $\mathbb{R}_{>0}$.
} 
In [12, Section 24], Hahn noted two somewhat obvious facts about the composition of comparison functions. Suppose $\alpha, \alpha_{1}, \alpha_{2} \in \mathcal{K}$ and $\sigma \in \mathcal{L}$. Then

$$
\begin{aligned}
& \alpha_{1} \circ \alpha_{2} \in \mathcal{K} ; \text { and } \\
& \alpha \circ \sigma \in \mathcal{L} .
\end{aligned}
$$

In addition, we observe that for $\sigma_{1}, \sigma_{2} \in \mathcal{L}$ and with $c \doteq \sigma_{1} \circ \sigma_{2}(0)$ we have

$$
\sigma_{1} \circ \sigma_{2}-c \in \mathcal{K}
$$

As a final definition, Hahn [12, Defn 24.2] introduced the class of $\mathcal{K} \mathcal{L}$ functions:

Definition 4 A function $\beta: \mathbb{R}_{\geq 0} \times \mathbb{R}_{\geq 0} \rightarrow \mathbb{R}_{\geq 0}$ is said to be of class- $\mathcal{K} \mathcal{L}(\beta \in \mathcal{K} \mathcal{L})$ if it is class- $\mathcal{K}$ in its first argument and class $-\mathcal{L}$ in its second argument. In other words, $\beta \in \mathcal{K} \mathcal{L}$ if for each fixed $t \in \mathbb{R}_{\geq 0}, \beta(\cdot, t) \in \mathcal{K}$ and for each fixed $s \in \mathbb{R}_{\geq 0}, \beta(s, \cdot) \in \mathcal{L}$.

For consistency, we will generally use $\alpha$ or $\varphi$ for functions of class- $\mathcal{K}$ or class$\mathcal{K}_{\infty}, \sigma$ for functions of class- $\mathcal{L}, \beta$ for functions of class- $\mathcal{K} \mathcal{L}, \rho$ for positive definite functions, and $a, b, c$, or $\lambda$ for positive constants in $\mathbb{R}_{>0}$. For functions $\alpha \in \mathcal{K}$ that are differentiable, we will denote the derivative by $\alpha^{\prime}$. We will denote the identity function by id; i.e., $\operatorname{id}(s)=s$ for all $s \in \mathbb{R}_{\geq 0}$.

\subsection{Example: Lyapunov functions and $\mathcal{K} \mathcal{L}$-stability}

As a brief example of the elegance enabled by the use of comparison functions, we demonstrate the proof that a Lyapunov function implies uniform global asymptotic stability of the origin for an autonomous system

$$
\dot{x}=f(x) \text {. }
$$

Here, we assume the existence and uniqueness of solutions and denote solutions to the above differential equation from an initial point $x_{0}=x(0) \in \mathbb{R}^{n}$ by $x(t)$ for all $t \in \mathbb{R}_{\geq 0}$.

Definition 5 The origin is globally asymptotically stable for $\dot{x}=f(x)$ if there exists a function $\beta \in \mathcal{K} \mathcal{L}$ such that, for all $x_{0} \in \mathbb{R}^{n}$,

$$
|x(t)| \leq \beta\left(\left|x_{0}\right|, t\right), \quad \forall t \in \mathbb{R}_{\geq 0} .
$$

It is obvious that the above definition is equivalent to the standard definition of global asymptotic stability as the combination of stability and attractivity (see [22, Proposition 2.5]).

Definition 6 A Lyapunov function $V: \mathbb{R}^{n} \rightarrow \mathbb{R}_{\geq 0}$ for $\dot{x}=f(x)$ is a continuously differentiable function such that there exist $\alpha_{1}, \alpha_{2} \in \mathcal{K}_{\infty}$, a continuous positive definite function $\rho: \mathbb{R}^{n} \rightarrow \mathbb{R}_{\geq 0}$ and, for all $x \in \mathbb{R}^{n}$,

$$
\alpha_{1}(|x|) \leq V(x) \leq \alpha_{2}(|x|)
$$




$$
\dot{V}(x)=\langle\nabla V(x), f(x)\rangle \leq-\rho(x) .
$$

Assume the existence of a Lyapunov function for $\dot{x}=f(x)$. Lemma 18 in the sequel yields the existence of $\alpha \in \mathcal{K}_{\infty}$ and $\sigma \in \mathcal{L}$ such that

$$
\rho(x) \geq \alpha(|x|) \sigma(|x|), \quad \forall x \in \mathbb{R}^{n} .
$$

Since the functions $\alpha_{1}, \alpha_{2} \in \mathcal{K}_{\infty}$, they are both invertible and we define

$$
\hat{\alpha} \doteq \alpha \circ \alpha_{2}^{-1} \in \mathcal{K}_{\infty} \quad \text { and } \quad \hat{\sigma} \doteq \sigma \circ \alpha_{1}^{-1} \in \mathcal{L} .
$$

Finally, we define $\hat{\rho}: \mathbb{R}_{\geq 0} \rightarrow \mathbb{R}_{\geq 0}$ by

$$
\hat{\rho}(s) \doteq \hat{\alpha}(s) \hat{\sigma}(s), \quad \forall s \in \mathbb{R}_{\geq 0}
$$

and note that the product of a class- $\mathcal{K}_{\infty}$ function and a class- $\mathcal{L}$ function is a positive definite function and hence $\hat{\rho}$ is a positive definite function. Therefore, using (1), (3), and the definitions of functions $\hat{\alpha}, \hat{\sigma}$, and $\hat{\rho}$, we may bound the decrease condition (2) as

$$
\dot{V}(x) \leq-\alpha(|x|) \sigma(|x|) \leq-\alpha\left(\alpha_{2}^{-1}(V(x))\right) \sigma\left(\alpha_{1}^{-1}(V(x))\right)=-\hat{\rho}(V(x)) .
$$

Using a comparison lemma from the sequel (Lemma 20) we have the existence of a function $\hat{\beta} \in \mathcal{K} \mathcal{L}$ such that, for all $x(0)=x_{0} \in \mathbb{R}^{n}$,

$$
V(x(t)) \leq \hat{\beta}\left(V\left(x_{0}\right), t\right), \quad \forall t \in \mathbb{R}_{\geq 0} .
$$

By manipulating the upper and lower bounds in (1) we obtain

$$
|x(t)| \leq \alpha_{1}^{-1}\left(\hat{\beta}\left(\alpha_{2}\left(\left|x_{0}\right|\right), t\right)\right) \doteq \beta\left(\left|x_{0}\right|, t\right),
$$

for all $t \in \mathbb{R}_{\geq 0}$ and $x_{0} \in \mathbb{R}^{n}$, proving global asymptotic stability of the origin.

Furthermore, we note that any nonlinear scaling of a Lyapunov function by a continuously differentiable function $\bar{\alpha} \in \mathcal{K}_{\infty}$ satisfying $\bar{\alpha}^{\prime}(s)>0$ for all $s \in \mathbb{R}_{>0}$ yields a Lyapunov function ${ }^{6}$. To see this, given a Lyapunov function $V$, functions $\alpha_{1}, \alpha_{2} \in \mathcal{K}_{\infty}$ and a continuous positive definite function $\rho: \mathbb{R}^{n} \rightarrow \mathbb{R}_{\geq 0}$ so that (1) and (2) hold, define

$$
W(x) \doteq \bar{\alpha}(V(x)), \quad \forall x \in \mathbb{R}^{n} .
$$

We define $\hat{\alpha}_{1} \doteq \bar{\alpha} \circ \alpha_{1} \in \mathcal{K}_{\infty}$ and $\hat{\alpha}_{2} \doteq \bar{\alpha} \circ \alpha_{2} \in \mathcal{K}_{\infty}$ and see that (1) implies that $W$ satisfies the necessary upper and lower bounds

$$
\hat{\alpha}_{1}(|x|) \leq W(x) \leq \hat{\alpha}_{2}(|x|), \quad \forall x \in \mathbb{R}^{n} .
$$

\footnotetext{
${ }^{6}$ Despite the fact that functions $\alpha \in \mathcal{K}$ are strictly increasing, it is possible that $\alpha^{\prime}(s)=0$ for isolated points $s \in \mathbb{R}_{>0}$. For example, $\alpha(s) \doteq \int_{0}^{s}(1+\sin (\tau)) d \tau$ is one such function.
} 
Since $\bar{\alpha} \in \mathcal{K}_{\infty}$ is continuously differentiable and $\bar{\alpha}^{\prime}(s)>0$ for all $s \in \mathbb{R}_{>0}$, we have

$$
\langle\nabla W(x), f(x)\rangle=\bar{\alpha}^{\prime}(V(x))\langle\nabla V(x), f(x)\rangle \leq-\bar{\alpha}^{\prime}(V(x)) \rho(x)
$$

for all $x \in \mathbb{R}^{n} \backslash\{0\}$, where the right-hand side is a continuous positive definite function. In other words, the function $W(x)=\bar{\alpha}(V(x))$ is a Lyapunov function for any continuously differentiable $\bar{\alpha} \in \mathcal{K}_{\infty}$.

Finally, let the continuous positive definite function $\hat{\rho}: \mathbb{R}_{\geq 0} \rightarrow \mathbb{R}_{\geq 0}$ be defined as in (4) so that we have the decrease condition (5). Then for $\hat{\rho}$, Lemma 24 in the sequel yields an $\bar{\alpha} \in \mathcal{K}_{\infty}$ that is continuously differentiable on $\mathbb{R}_{>0}$ such that

$$
\bar{\alpha}(s) \leq \hat{\rho}(s) \bar{\alpha}^{\prime}(s), \quad \forall s \in \mathbb{R}_{>0}
$$

Repeating the calculations of (5) and (6) we see that, for $W(x) \doteq \bar{\alpha}(V(x))$ we have

$$
\dot{W}(x)=\langle\nabla W(x), f(x)\rangle \leq-\bar{\alpha}^{\prime}(V(x)) \hat{\rho}(x) \leq-\bar{\alpha}(V(x))=-W(x)
$$

for all $x \in \mathbb{R}^{n} \backslash\{0\}$. In other words, the Lyapunov function $W(x)=\bar{\alpha}(V(x))$ decreases exponentially.

\subsection{A word on regularity}

Since class- $\mathcal{K}$ and class- $\mathcal{L}$ functions are monotonic, a theorem due to Lebesgue states that these functions must be differentiable almost everywhere (see, for example, [31, Section 2]) and this property holds without the functions necessarily being continuous.

While we have assumed that functions of class- $\mathcal{K}$ or class- $\mathcal{L}$ are continuous functions, it is sometimes useful to require functions with more regularity; for example, when discussing the nonlinear scaling of Lyapunov functions in the previous section we required the nonlinear scaling $\alpha \in \mathcal{K}_{\infty}$ to be continuously differentiable. In fact, we can always bound comparison functions from above and below by smooth functions on $\mathbb{R}_{>0}$. We here state [5, Lemma 2.5] as two lemmas to separately state the results for nondecreasing and nonincreasing functions.

Lemma 1 Let $\alpha: \mathbb{R}_{\geq 0} \rightarrow \mathbb{R}_{\geq 0}$ be continuous, zero at zero, strictly positive on $\mathbb{R}_{>0}$, and nondecreasing. Then there exist functions $\alpha_{1}, \alpha_{2} \in \mathcal{K}$, smooth on $\mathbb{R}_{>0}$, so that

$$
\alpha_{1}(s) \leq \alpha(s) \leq \alpha_{2}(s), \quad \forall s \in \mathbb{R}_{\geq 0}
$$

Furthermore, if $\lim _{s \rightarrow \infty} \alpha(s)=\infty$ then both functions may be chosen to satisfy $\alpha_{1}, \alpha_{2} \in \mathcal{K}_{\infty}$.

Lemma 2 Let $\sigma: \mathbb{R}_{\geq 0} \rightarrow \mathbb{R}_{>0}$ be continuous, nonincreasing, and satisfying $\lim _{s \rightarrow \infty} \sigma(s)=0$. Then, there exist functions $\sigma_{1}, \sigma_{2} \in \mathcal{L}$, smooth on $\mathbb{R}_{>0}$, so that

$$
\sigma_{1}(s) \leq \sigma(s) \leq \sigma_{2}(s), \quad \forall s \in \mathbb{R}_{\geq 0}
$$


In contrast to the above results, when we assume that the function we wish to approximate is of class- $\mathcal{K}$ or class- $\mathcal{L}$, we may additionally control how close the smooth function is to the given function.

Lemma 3 For $\alpha \in \mathcal{K}$ and any $\varepsilon>0$ there exist $\alpha_{1}, \alpha_{2} \in \mathcal{K}$, smooth on $\mathbb{R}_{>0}$, so that, for all $s \in \mathbb{R}_{\geq 0}$

$$
\alpha(s)-\varepsilon<\alpha_{1}(s) \leq \alpha(s) \leq \alpha_{2}(s)<\alpha(s)+\varepsilon
$$

Lemma 4 For $\sigma \in \mathcal{L}$ and any $\varepsilon>0$ there exist $\sigma_{1}, \sigma_{2} \in \mathcal{K}_{\infty}$, smooth on $\mathbb{R}_{>0}$ so that, for all $s \in \mathbb{R}_{\geq 0}$

$$
\sigma(s)-\varepsilon<\sigma_{1}(s) \leq \sigma(s) \leq \sigma_{2}(s) \leq \sigma(s)+\varepsilon
$$

With a further requirement that the given function be of class- $\mathcal{K}_{\infty}$, we obtain the following two smooth approximation lemmas.

Lemma 5 [9, Lemma B.2.1] Given $\alpha \in \mathcal{K}_{\infty}$ and $\rho: \mathbb{R}_{\geq 0} \rightarrow \mathbb{R}_{\geq 0}$ is continuous and positive definite, there exists $\tilde{\alpha} \in \mathcal{K}_{\infty}$, smooth on $\mathbb{R}_{>0}$, so that

$$
|\alpha(s)-\tilde{\alpha}(s)| \leq \rho(\alpha(s)), \quad \forall s \in \mathbb{R}_{\geq 0}
$$

Lemma 6 [9, Lemma B.2.2] Given $\alpha \in \mathcal{K}_{\infty}$ and $\rho: \mathbb{R}_{\geq 0} \rightarrow \mathbb{R}_{\geq 0}$ is continuous and positive definite, there exists $\alpha_{1}, \alpha_{2} \in \mathcal{K}_{\infty}$, smooth on $\mathbb{R}_{>0}$, so that, for all $s \in \mathbb{R}_{\geq 0}$,

$$
\alpha(s)-\rho(\alpha(s)) \leq \alpha_{1}(s)<\alpha(s)<\alpha_{2}(s) \leq \alpha(s)+\rho(\alpha(s)) .
$$

The proofs of all of the lemmas in this section follow a similar idea where a continuous piecewise linear function is constructed to bound the given function from above or below while giving the additional desired inequalities. Standard techniques can then be used to smooth these functions where they are pieced together. As an example, and because we have not seen the result previously in the literature, we present the proof of Lemma 3 in Appendix A.1 and provide a remark on the necessary modifications to obtain Lemma 4.

\section{Upper bounds}

Arguably the most useful lemma regarding $\mathcal{K} \mathcal{L}$-functions is widely known as Sontag's Lemma on $\mathcal{K} \mathcal{L}$-Estimates which originally appeared as [36, Proposition 7]. The version given here is from [19] and provides some nice properties on one of the resulting $\mathcal{K}_{\infty}$ functions. The proof is provided in Appendix A.2.

Lemma 7 [19, Lemma 5.3] Given $\beta \in \mathcal{K} \mathcal{L}$ and $\lambda \in \mathbb{R}_{>0}$, there exist $\alpha_{1}, \alpha_{2} \in \mathcal{K}_{\infty}$ such that $\alpha_{1}(\cdot)$ is Lipschitz on its domain, smooth on $\mathbb{R}_{>0}, \alpha_{1}(s) \leq s \alpha_{1}^{\prime}(s)$ for all $s \in \mathbb{R}_{>0}$ and

$$
\alpha_{1}(\beta(s, t)) \leq \alpha_{2}(s) e^{-\lambda t}, \quad \forall(s, t) \in \mathbb{R}_{\geq 0} \times \mathbb{R}_{\geq 0}
$$


A precursor to the above result was presented by Hahn [12]:

Lemma 8 Let $\alpha_{1}, \alpha_{2} \in \mathcal{K}, \sigma \in \mathcal{L}$, and either $\alpha_{1}^{\prime}$ or $\alpha_{2}$ be bounded. Then, there exist $\hat{\alpha} \in \mathcal{K}$ and $\hat{\sigma} \in \mathcal{L}$ such that

$$
\alpha_{1}\left(\alpha_{2}(s) \sigma(t)\right) \leq \hat{\alpha}(s) \hat{\sigma}(t), \quad \forall s, t \in \mathbb{R}_{\geq 0}
$$

Proof If there exists a $c \in \mathbb{R}_{>0}$ so that $\alpha_{1}^{\prime}(s) \leq c$ for all $s \in \mathbb{R}_{>0}$, then the mean value theorem yields the desired result with $\hat{\alpha} \doteq c \alpha_{2}$ and $\hat{\sigma} \doteq \sigma$.

On the other hand, if there exists $c \in \mathbb{R}_{>0}$ so that $\alpha_{2}(s) \leq c$ for all $s \in \mathbb{R}_{>0}$, then the result holds with $\hat{\alpha}(s) \doteq \sqrt{\alpha_{1}\left(\sigma(0) \alpha_{2}(s)\right)}$ for all $s \in \mathbb{R}_{\geq 0}$ and $\hat{\sigma}(t) \doteq \sqrt{\alpha_{1}(c \sigma(t))}$ for all $t \in \mathbb{R}_{\geq 0}$.

The following result describes a class of functions that can be bounded from above by a class- $\mathcal{K} \mathcal{L}$ function. This result was proved, though not formally stated, in [22].

Lemma 9 [1, Lemma 4.1] Suppose a function $\phi: \mathbb{R}_{\geq 0} \times \mathbb{R}_{\geq 0} \rightarrow \mathbb{R}$ satisfies

- for all $r, \varepsilon \in \mathbb{R}_{>0}$, there exists some $T=T(r, \varepsilon) \in \mathbb{R}_{>0}$ such that $\phi(s, t)<\varepsilon$ for all $s \leq r$ and $t \geq T$; and

- for all $\varepsilon \in \mathbb{R}_{>0}$ there exists $\delta \in \mathbb{R}_{>0}$ such that $\phi(s, t) \leq \varepsilon$ for all $s \leq \delta$ and all $t \in \mathbb{R}_{\geq 0}$.

Then there exists some $\beta \in \mathcal{K} \mathcal{L}$ such that $\phi(s, t) \leq \beta(s, t)$ for all $s, t \in \mathbb{R}_{\geq 0}$.

As in analysis more generally, the existence of a triangle inequality is extremely useful. In [35, Equation (12)], Sontag observed that for any function $\alpha \in \mathcal{K}$ and any $a, b \in \mathbb{R}_{\geq 0}$,

$$
\alpha(a+b) \leq \alpha(2 a)+\alpha(2 b)
$$

A generalization of this was presented in [17] where an additional function $\varphi \in \mathcal{K}_{\infty}$ can be used to trade off the relative weighting on the first argument versus the second argument. The following is sometimes referred to as a weak triangle inequality. A proof is provided in Appendix A.3.

Lemma 10 [17, Equation (6)] Given $\alpha \in \mathcal{K}$ and any function $\varphi \in \mathcal{K}_{\infty}$ such that $\varphi-\mathrm{id} \in \mathcal{K}_{\infty}$, then for any $a, b \in \mathbb{R}_{\geq 0}$

$$
\alpha(a+b) \leq \alpha(\varphi(a))+\alpha\left(\varphi \circ(\varphi-\mathrm{id})^{-1}(b)\right) .
$$

Clearly, choosing $\varphi(s)=2 s$ for all $s \in \mathbb{R}_{\geq 0}$ regains the original form of the weak triangle inequality (8). More generally, we can select $\varphi(s)=(1+c) s$ with $c \in \mathbb{R}_{>0}$, which satisfies

$$
\varphi \circ(\varphi-\mathrm{id})^{-1}(s)=\frac{1+c}{c} s
$$


Consequently, choosing $c<1$ puts a larger weighting on the $b$ term, whereas choosing $c>1$ puts a larger weighting on the $a$ term.

We note that an alternate, more symmetric, form of (9) can be obtained by choosing $\hat{\varphi} \doteq \varphi-\mathrm{id} \in \mathcal{K}_{\infty}$ so that

$$
\alpha(a+b) \leq \alpha \circ(\hat{\varphi}+\mathrm{id})(a)+\alpha \circ\left(\hat{\varphi}^{-1}+\mathrm{id}\right)(b), \quad \forall a, b \in \mathbb{R}_{\geq 0}
$$

This form of the weak triangle inequality was presented in [33, Lemma 1.1.2].

It is possible to weakly factor functions of two variables that are jointly of class- $\mathcal{K}$.

Lemma 11 [3, Corollary IV.5] Suppose $\alpha: \mathbb{R}_{\geq 0}^{2} \rightarrow \mathbb{R}_{\geq 0}$ is such that $\alpha(\cdot, s) \in \mathcal{K}$ for each fixed $s \in \mathbb{R}_{\geq 0}$ and $\alpha(r, \cdot) \in \mathcal{K}$ for each fixed $r \in \mathbb{R}_{\geq 0}$. Then there exists a function $\hat{\alpha} \in \mathcal{K}$ such that

$$
\alpha(r, s) \leq \hat{\alpha}(r) \hat{\alpha}(s)
$$

We present a sketch of the proof in Sect. 7.4.

A precursor to the above appeared as [36, Corollary 10] where the relationship between the arguments is a product.

Lemma 12 [36, Corollary 10] Given $\alpha \in \mathcal{K}_{\infty}$ there exist $\alpha_{1}, \alpha_{2} \in \mathcal{K}_{\infty}$ such that

$$
\alpha(r s) \leq \alpha_{1}(r) \alpha_{2}(s), \quad \forall r, s \in \mathbb{R}_{\geq 0} .
$$

Hahn [12] made use of a similar upper bound in a fairly simple case. In particular, given $\alpha \in \mathcal{K}$ and constants $c, \lambda \in \mathbb{R}_{>0}$ such that $\alpha(s) \leq c s^{\lambda}$ for all $s \in \mathbb{R}_{\geq 0}$. Then

$$
\alpha(r s) \leq c r^{\lambda} s^{\lambda}, \quad \forall r, s \in \mathbb{R}_{\geq 0}
$$

The following lemma provides an upper bound on a given class- $\mathcal{K}$ function in terms of the composition of convex and concave functions of class- $\mathcal{K}$.

Lemma 13 [30, Lemma 14] Given $\alpha \in \mathcal{K}$ there exist continuously differentiable and convex $\alpha_{v} \in \mathcal{K}$ and continuously differentiable and concave $\alpha_{c} \in \mathcal{K}$ such that

$$
\alpha(s) \leq \alpha_{c} \circ \alpha_{v}(s), \quad \forall s \in \mathbb{R}_{\geq 0}
$$

The key idea of the proof involves the fact that the integral of a class- $\mathcal{K}$ function is convex. On the one hand, the convex function required is directly defined by

$$
\alpha_{v}(s) \doteq \frac{\alpha^{-1}\left(s^{*}\right)}{s^{*}} \int_{0}^{2 s} \alpha(\tau) d \tau+s, \quad \forall s \in \mathbb{R}_{\geq 0}
$$


where $s^{*} \doteq \min \left\{1, \frac{a}{2}\right\}$ and $a=\lim _{s \rightarrow \infty} \alpha(s)$. These latter points guarantee that the inverse $\alpha^{-1}$ is well defined. On the other hand, the concave function is defined by specifying its inverse as the convex function

$$
\alpha_{c}^{-1}(s) \doteq \begin{cases}\int_{0}^{s} \alpha^{-1}(\tau) d \tau, & \forall s \in\left[0, s^{*}\right] \\ \alpha_{c}^{-1}\left(s^{*}\right)+\left(s-s^{*}\right) \alpha^{-1}\left(s^{*}\right), & \forall s>s^{*} .\end{cases}
$$

An upper bound for class- $\mathcal{L}$ functions was presented in [12] as follows:

Lemma 14 If $\sigma \in \mathcal{L}$ then there exist $\sigma_{1}, \sigma_{2} \in \mathcal{L}$ such that

$$
\sigma(r+s) \leq \sigma_{1}(r) \sigma_{2}(s), \quad \forall r, s \in \mathbb{R}_{\geq 0}
$$

The proof of the above is provided in Appendix A.4.

Finally, we introduce the Legendre-Fenchel transform to present a general version of Young's Inequality. Suppose $\alpha \in \mathcal{K}_{\infty}$ is continuously differentiable and that, in addition, $\alpha^{\prime} \in \mathcal{K}_{\infty}$. To ease the notation, denote $\left(\alpha^{\prime}\right)^{-1}(s) \doteq \varphi(s)$ for all $s \in \mathbb{R}_{\geq 0}$. Then the Legendre-Fenchel transform of $\alpha$ is defined by

$$
\ell \alpha(s) \doteq \int_{0}^{s} \varphi(\tau) d \tau, \quad \forall s \in \mathbb{R}_{\geq 0}
$$

The Legendre-Fenchel transform appears to have first been used in conjunction with comparison functions in [29]. Several interesting properties of the Legendre-Fenchel transform were provided in [20].

Lemma 15 [20, Lemma A.1] If $\alpha, \alpha^{\prime} \in \mathcal{K}_{\infty}$, then the Legendre-Fenchel transform (12) satisfies

(i) $\ell \alpha(s)=s \varphi(s)-\alpha \circ \varphi(s), \forall s \in \mathbb{R}_{\geq 0}$;

(ii) $\ell \ell \alpha(s)=\alpha(s), \quad \forall s \in \mathbb{R}_{\geq 0}$;

(iii) $\ell \alpha\left(\alpha^{\prime}(s)\right)=s \alpha^{\prime}(s)-\alpha(s), \forall s \in \mathbb{R}_{\geq 0}$; and

(iv) $\ell \alpha \in \mathcal{K}_{\infty}$; .

Using the Legendre-Fenchel transform, the following general version of Young's Inequality was presented in [29].

Lemma 16 For any continuously differentiable $\alpha \in \mathcal{K}_{\infty}$ such that $\alpha^{\prime} \in \mathcal{K}_{\infty}$ and any $x, y \in \mathbb{R}^{n}$

$$
x^{T} y \leq \alpha(|x|)+\ell \alpha(|y|)
$$

with equality if and only if

$$
y=\frac{\alpha^{\prime}(|x|)}{|x|} x .
$$


The original version of Young's inequality [13, Theorem 156] bounds the product of two nonnegative numbers as follows: for any $\hat{\alpha} \in \mathcal{K}_{\infty}$ and $a, b \in \mathbb{R}_{\geq 0}$,

$$
a b \leq \int_{0}^{a} \hat{\alpha}(\tau) d \tau+\int_{0}^{b} \hat{\alpha}^{-1}(\tau) d \tau
$$

with equality if and only if $b=\hat{\alpha}(a)$. The relationship of (13) to Lemma 16 is straightforward to see by considering $\hat{\alpha}=\alpha^{\prime}$.

A useful result for bounding from above the product of two nonnegative numbers based on any given $\mathcal{K}_{\infty}$ function was presented in [30].

Lemma 17 For any $\alpha \in \mathcal{K}_{\infty}$ and any $p \in \mathbb{R}_{>0}$,

$$
a b \leq \alpha^{-1}(p a) a+\frac{\alpha(b) b}{p}, \quad \forall a, b \in \mathbb{R}_{\geq 0} .
$$

The proof is straightforward by considering the two cases of $\alpha^{-1}(p a) \geq b$ and $\alpha^{-1}(p a)<b$. Alternatively, this can be shown via Young's inequality (13) by considering $\alpha \doteq \frac{1}{p} \hat{\alpha} \in \mathcal{K}_{\infty}$.

\section{Lower bounds}

As comparison functions are most frequently used in gain and stability estimates, they tend to be used to upper bound various quantities. As a consequence, the literature contains many more results for bounding functions from above than for bounding functions from below. Nonetheless, lower bounds have occasionally proved useful.

As previously noted, the initial use of class- $\mathcal{K}$ functions was for the characterization of locally positive definite functions. However, it is not possible to bound every globally positive definite function from below by a function of class- $\mathcal{K}$. A complete characterization of positive definite functions on $\mathbb{R}_{\geq 0}$ in terms of a lower bound depending on both a class- $\mathcal{K}$ function and a class- $\mathcal{L}$ function was given by in [3, Lemma IV.1]. The version presented here extends the characterization to positive definite functions defined on $\mathbb{R}^{n}$. A proof is provided in Appendix A.5.

Lemma 18 Let $\rho: \mathbb{R}^{n} \rightarrow \mathbb{R}_{\geq 0}$ be a continuous positive definite function. Then, there exist functions $\alpha \in \mathcal{K}_{\infty}$ and $\sigma \in \mathcal{L}$ such that

$$
\rho(x) \geq \alpha(|x|) \sigma(|x|), \quad \forall x \in \mathbb{R}^{n} .
$$

The following lower bound on functions of class- $\mathcal{K} \mathcal{L}$ was introduced in [37] to separate the effect of the two arguments when considering the decrease condition for an input-to-output stability Lyapunov function. ${ }^{7}$ The main idea of the proof is discussed in Sect. 7.4.

\footnotetext{
7 We note that the statement of Lemma 19 in [37] was not entirely precise in two respects. The first is that the authors explicitly defined class- $\mathcal{K} \mathcal{L}$ functions as nonincreasing, rather than strictly decreasing, in their
} 
Lemma 19 [37, Lemma A.2] For any $\beta \in \mathcal{K} \mathcal{L}$ there exist $\alpha_{1} \in \mathcal{K}, \alpha_{2} \in \mathcal{K}_{\infty}$ such that

$$
\beta(s, t) \geq \frac{\alpha_{1}(s)}{1+\alpha_{2}(t)}
$$

for all $(s, t) \in \mathbb{R}_{\geq 0} \times \mathbb{R}_{\geq 0}$.

\section{The comparison principle}

The comparison principle or comparison lemma makes use of a (usually solvable) scalar differential inequality to make statements about the nature of solutions to a scalar differential equation. An example application of this principle was provided in Sect. 2.1. In the case where the right-hand side of the scalar differential inequality is a continuous negative definite function, then solutions are bounded by a function of class- $\mathcal{K} \mathcal{L}$. The following result was first demonstrated in a slightly more restrictive form by Hahn [12, Section 24E].

Lemma 20 [37, Lemma A.4] For any continuous positive definite function $\rho: \mathbb{R}_{\geq 0} \rightarrow$ $\mathbb{R}_{\geq 0}$ there exists $\beta \in \mathcal{K} \mathcal{L}$ such that if $y(\cdot)$ is any locally absolutely continuous function defined on some interval $[0, T]$ with $y(t) \geq 0$ for all $t \in[0, T]$, and if $y(\cdot)$ satisfies the differential inequality

$$
\dot{y}(t) \leq-\lambda \rho(y(t))
$$

for almost all $t \in[0, T]$ for some $\lambda \geq 0$ with $y(0)=y_{0} \in \mathbb{R}_{\geq 0}$ then

$$
y(t) \leq \beta\left(y_{0}, \lambda t\right), \quad \forall t \in[0, T] .
$$

The above, with $\lambda=1$, first appeared in [22, Lemma 4.4]. A precursor to this where $y(t)$ satisfying a differential equation, rather than the inequality above, implies a $\mathcal{K} \mathcal{L}$ bound appeared in [35, Lemma 6.1]. A proof of Lemma 20 is provided in Appendix A.6.

An extension to the above comparison principle allows the right-hand side of the differential inequality to also depend on an external input. This is particularly useful when considering robust stability in the context of input-to-state stability and its variants. The following two lemmas allow the input to enter via a maximization and a summation, respectively.

Lemma 21 [3, Lemma IV.2] Given any continuous positive definite function $\rho$ : $\mathbb{R}_{\geq 0} \rightarrow \mathbb{R}_{\geq 0}$ there exists $\beta \in \mathcal{K} \mathcal{L}$ so that if, for some $\bar{t} \in \mathbb{R}_{>0}, v:[0, \bar{t}) \rightarrow \mathbb{R}_{\geq 0}$ is a

Footnote 7 continued

second argument. However, this definition admits functions such that $\beta(s, t)=0$ for some $s, t \in \mathbb{R}_{>0}$ finite, while the right-hand side of (14) is strictly positive. The second is that it is necessary that $\alpha_{2} \in \mathcal{K}_{\infty}$ whereas in [37, Lemma A.2] it is only stated that $\alpha_{2} \in \mathcal{K}$. However, if $\alpha_{2} \in \mathcal{K}$ is not of class- $\mathcal{K}_{\infty}$, then the right-hand side of (14) may remain bounded away from zero as $t \rightarrow \infty$ while $\lim _{t \rightarrow \infty} \beta(s, t)=0$. 
continuous function and $y:[0, \bar{t}) \rightarrow \mathbb{R}$ is a (locally) absolutely continuous function with $y(0)=y_{0} \in \mathbb{R}_{\geq 0}$, and if

$$
\dot{y}(t) \leq-\rho(\max \{y(t)+v(t), 0\})
$$

holds for almost all $t \in[0, \bar{t})$ then

$$
y(t) \leq \max \left\{\beta\left(y_{0}, t\right), \sup _{\tau \in[0, t]}|v(\tau)|\right\}, \quad \forall t \in[0, \bar{t}) .
$$

The proof relies on Lemma 18 applied to the positive definite function $\rho$ to obtain $\alpha \in \mathcal{K}_{\infty}$ and $\sigma \in \mathcal{L}$, so that, with the decreasing property of $\sigma, \rho(s) \geq \alpha(s) \sigma(2 s)$ for all $s \in \mathbb{R}_{\geq 0}$. Without being overly precise, two time intervals are then considered: times such that $y(t) \leq \sup _{\tau \in[0, t]}|v(\tau)|$ and times such that $y(t)$ exceeds this bound. The former set is invariant since $y(t)$ is decreasing and $\sup _{\tau \in[0, t]}|v(\tau)|$ is increasing. In the latter case, we see that $y(t)>v(t)$ so that $y(t) \leq y(t)+v(t) \leq 2 y(t)$. Then, since $\alpha \in \mathcal{K}_{\infty}, \dot{y}(t) \leq \alpha(y(t)) \sigma(2 y(t))$ and we can appeal to Lemma 20 to obtain the desired result. See [3] for a detailed proof.

Lemma 22 [3, Corollary IV.3] Given any continuous positive definite function $\rho$ : $\mathbb{R}_{\geq 0} \rightarrow \mathbb{R}_{\geq 0}$ there exists $\beta \in \mathcal{K} \mathcal{L}$ so that if, for some $\bar{t} \in \mathbb{R}_{>0}, v:[0, \bar{t}) \rightarrow \mathbb{R}_{\geq 0}$ is a measureable, locally essentially bounded function and $y:[0, \bar{t}) \rightarrow \mathbb{R}_{\geq 0}$ is (locally) absolutely continuous function with $y(0)=y_{0} \in \mathbb{R}_{\geq 0}$, and if

$$
\dot{y}(t) \leq-\rho(y(t))+v(t)
$$

for almost all $t \in[0, \bar{t})$, then

$$
y(t) \leq \beta\left(y_{0}, t\right)+\int_{0}^{t} 2 v(\tau) d \tau, \quad \forall t \in[0, \bar{t}) .
$$

The proof follows by defining certain auxiliary functions and showing that these functions satisfy the requirements of Lemma 21. In particular, let $w(t)$ be the solution to the initial value problem

$$
\dot{w}(t)=-\rho(w(t))+v(t), \quad w(0)=y(0),
$$

let $v_{1}(t)=\int_{0}^{t} v(\tau) d \tau$, and let $w_{1}(t)=w(t)-v_{1}(t)$. The function $w_{1}$ then plays the role of $y$ in Lemma 21. As before, see [3] for a detailed proof.

\section{Other relationships}

In addition to the previously presented lemmas providing upper and lower bounds and comparison principles, several results involving comparison functions are available 
that do not neatly fit into these categories. In this section, we summarize several such results.

Many factorizations of comparison functions are possible, with the derivations frequently relying on the invertibility of class- $\mathcal{K}_{\infty}$ functions. A proof of the following is in Appendix A.7.

Lemma 23 [33, Lemma 1.1.3] For any $\alpha \in \mathcal{K}$, there exist $\alpha_{1}, \alpha_{2} \in \mathcal{K}$ such that

$$
\alpha+\mathrm{id}=\left(\alpha_{1}+\mathrm{id}\right) \circ\left(\alpha_{2}+\mathrm{id}\right) .
$$

Furthermore, if $\alpha \in \mathcal{K}_{\infty}$ then $\alpha_{1}$ and $\alpha_{2}$ can be chosen of class- $\mathcal{K}_{\infty}$ as well.

The next two lemmas presented were used to modify decrease conditions of Lyapunov functions in continuous time (Lemma 24) and discrete time (Lemma 25). The following is a combination of [30, Lemmas 11 and 12].

Lemma 24 [19, Lemma 5.4] For each continuous, positive definite function $\rho$ : $\mathbb{R}_{\geq 0} \rightarrow \mathbb{R}_{\geq 0}$ there exists $\alpha \in \mathcal{K}_{\infty}$ such that $\alpha(\cdot)$ is locally Lipschitz on its domain, continuously differentiable on $(0, \infty)$ and

$$
\alpha(s) \leq \rho(s) \alpha^{\prime}(s), \quad \forall s \in \mathbb{R}_{>0}
$$

The form of the function $\alpha \in \mathcal{K}_{\infty}$ used in the proof of Lemma 24 is

$$
\alpha(s) \doteq \exp \left(2 \int_{1}^{s} \frac{1}{\min \{\tau, \rho(\tau)\}} d \tau\right), \quad \forall s \in \mathbb{R}_{>0}
$$

and $\alpha(0)=0$.

Lemma 25 [18, Lemma 19] If $\lambda>1$ and if $\alpha \in \mathcal{K}_{\infty}$ satisfies $(\alpha-\mathrm{id}) \in \mathcal{K}_{\infty}$, then there exists $\hat{\alpha} \in \mathcal{K}_{\infty}$ such that

$$
\hat{\alpha} \circ \alpha(s)=\lambda \hat{\alpha}(s), \quad \forall s \in \mathbb{R}_{\geq 0}
$$

A proof of the above lemma is provided in Appendix A.8. While the proof is generally nonconstructive, for linear functions of class- $\mathcal{K}_{\infty}$

$$
\alpha(s)=c s, \quad c>1
$$

one choice for the $\mathcal{K}_{\infty}$ function of Lemma 25 can be seen to be

$$
\hat{\alpha}(s)=s^{m}, \quad m=\frac{\log \lambda}{\log c} .
$$

Since $\lambda, c>1$, we have that $m>0$ and

$$
\hat{\alpha} \circ \alpha(s)=c^{\frac{\log \lambda}{\log c}} s^{m}=\lambda s^{m}=\lambda \hat{\alpha}(s) .
$$


It is possible to derive a corollary to Lemma 25 to allow for positive constants less than one. The proof is provided in Appendix A.9.

Corollary 1 If $\lambda \in(0,1)$ and if $\alpha \in \mathcal{K}_{\infty}$ satisfies (id $\left.-\alpha\right) \in \mathcal{K}_{\infty}$ then there exists $\hat{\alpha} \in \mathcal{K}_{\infty}$ such that

$$
\hat{\alpha} \circ(\operatorname{id}-\alpha)(s)=\lambda \hat{\alpha}(s), \quad \forall s \in \mathbb{R}_{\geq 0} .
$$

Given a function of class- $\mathcal{K}$, it is possible to find another function of class- $\mathcal{K}$ that upper bounds the given function away from the origin and is linear near the origin.

Lemma $26\left[16\right.$, Lemma 1] For any $\alpha \in \mathcal{K}$ and any $\lambda \in \mathbb{R}_{>0}$, there exist two numbers $\lambda_{1}, \lambda_{2} \in \mathbb{R}_{>0}$ and a smooth $\hat{\alpha} \in \mathcal{K}_{\infty}$ such that

$$
\begin{aligned}
& \alpha(s) \leq \hat{\alpha}(s), \quad \forall s \geq \lambda \\
& \hat{\alpha}(s)=\lambda_{1} s, \quad \forall s \in\left[0, \lambda_{2}\right) .
\end{aligned}
$$

The following lemma describes a condition under which the order of composition of two class- $\mathcal{K}$ functions can be reversed whilst maintaining a contraction principle.

Lemma $27\left[38\right.$, Fact A.2] Let $\alpha_{1}, \alpha_{2} \in \mathcal{K}$ and let $c \in \mathbb{R}_{>0}$. Then

$$
\alpha_{1} \circ \alpha_{2}(s) \leq s, \quad \forall s \in[0, c] \Longleftrightarrow \alpha_{2} \circ \alpha_{1}(s) \leq s, \quad \forall s \in\left[0, \alpha_{2}(c)\right]
$$

We note that [38, Fact A.2] only proved one direction of the implication. The proof of Lemma 27 is provided in Appendix A.10.

As a corollary to the above, we obtain the following result when both functions are of class- $\mathcal{K}_{\infty}$.

Corollary 2 Let $\alpha_{1}, \alpha_{2} \in \mathcal{K}_{\infty}$. Then

$$
\alpha_{1} \circ \alpha_{2}(s) \leq s, \quad \forall s \in \mathbb{R}_{\geq 0} \Longleftrightarrow \alpha_{2} \circ \alpha_{1}(s) \leq s \quad \forall s \in \mathbb{R}_{\geq 0}
$$

A simple example shows that, for more than two functions, we cannot change the order of composition and maintain a contraction condition. For all $s \in \mathbb{R}_{\geq 0}$, let

$$
\alpha_{1}(s) \doteq \sqrt{s}, \quad \alpha_{2}(s) \doteq \frac{3}{2} s, \quad \text { and } \quad \alpha_{3}(s) \doteq \frac{1}{2} s^{2}
$$

Then, for all $s \in \mathbb{R}_{>0}, \alpha_{1} \circ \alpha_{2} \circ \alpha_{3}(s)=\frac{\sqrt{3}}{2} s<s$ but $\alpha_{3} \circ \alpha_{2} \circ \alpha_{1}(s)=\frac{9}{8} s>s$.

Finally, in [23], the following characterization of growth rates of some class- $\mathcal{K}_{\infty}$ functions was provided.

Lemma 28 [23, Proposition 5] The following are equivalent:

- A function $\alpha \in \mathcal{K}_{\infty}$ satisfies, for every $\varepsilon \in \mathbb{R}_{>0}$

$$
\lim _{s \rightarrow \infty}\{\alpha((1+\varepsilon) s)-\alpha(s)\}=\infty
$$


- For every $\varepsilon \in \mathbb{R}_{>0}$ there exists $\varphi_{\varepsilon} \in \mathcal{K}_{\infty}$ such that

$$
\alpha(s-t) \leq \alpha((1+\varepsilon) s)-\varphi_{\varepsilon}(t), \quad \forall s \geq t \geq 0 .
$$

It was noted in [23] that $\mathcal{K}_{\infty}$ functions of the form $\alpha(s)=s^{\lambda}$ for $\lambda \in \mathbb{R}_{>0}$ satisfy the growth condition of (19). By contrast, $\mathcal{K}_{\infty}$ functions of the form $\alpha(s)=\lambda \log (1+s)$ for $\lambda \in \mathbb{R}_{>0}$ do not satisfy (19).

\section{Other function classes}

Functions of class- $\mathcal{K}, \mathcal{K}_{\infty}, \mathcal{L}$, and $\mathcal{K} \mathcal{L}$ have been used in stability theory for over 40 years going back to the original work of Massera and Hahn. Other useful classes have been introduced more recently.

\subsection{Functions of class- $\mathcal{K} \mathcal{L} \mathcal{D}$}

In [9], Grüne defined a subset of class- $\mathcal{K} \mathcal{L}$ functions, which he called class- $\mathcal{K} \mathcal{L} \mathcal{D}$.

Definition 7 A function $\mu: \mathbb{R}_{\geq 0} \times \mathbb{R} \rightarrow \mathbb{R}_{\geq 0}$ is said to be of class- $\mathcal{K} \mathcal{L D}$ if its restriction to $\mathbb{R}_{\geq 0} \times \mathbb{R}_{\geq 0}$ is class- $\mathcal{K} \mathcal{L}$ and, in addition,

$$
\mu(r, 0)=r \quad \text { and } \mu(\mu(r, t), s)=\mu(r, t+s)
$$

for all $r \in \mathbb{R}_{\geq 0}, s, t \in \mathbb{R}$.

The $\mathcal{D}$ in $\mathcal{K} \mathcal{L} \mathcal{D}$ above denotes dynamical and refers to the fact that, as a consequence of (20), a function of class- $\mathcal{K} \mathcal{L} \mathcal{D}$ defines a dynamical system on $\mathbb{R}_{\geq 0}$. Class$\mathcal{K} \mathcal{L D}$ functions were introduced in $[9,10]$ to characterize the notion of input-to-state dynamical stability (ISDS). ISDS is a robust stability concept equivalent to input-tostate stability and, as such, it is useful to know that any function of class- $\mathcal{K} \mathcal{L}$ can be bounded from above by a function of class- $\mathcal{K} \mathcal{L} \mathcal{D}$ as follows.

Lemma 29 [9, Lemma B.1.4] For any $\beta \in \mathcal{K} \mathcal{L}$, there exist functions $\mu \in \mathcal{K} \mathcal{L} \mathcal{D}$ and $\alpha \in \mathcal{K}_{\infty}$ such that

$$
\beta(s, t) \leq \mu(\alpha(s), t), \quad \forall s, t \in \mathbb{R}_{\geq 0}
$$

\subsection{Extended real-valued functions}

As was earlier noted, functions of class- $\mathcal{K}$ are invertible only on their range. Hence, when using functions of class- $\mathcal{K}$, if their inverses are required, a certain level of notational overhead is necessary. To reduce this notational burden and to provide approximate inverses for functions that are not strictly increasing (see class- $\mathcal{G}$ functions below), Ito [14] considered extended real-valued functions (see also [15]); that is, functions on $\overline{\mathbb{R}}_{\geq 0} \doteq \mathbb{R}_{\geq 0} \cup\{\infty\}$. 
For continuity at infinity of a function $\alpha: \overline{\mathbb{R}}_{\geq 0} \rightarrow \overline{\mathbb{R}}_{\geq 0}$ we intend that the usual limit condition $s_{n} \rightarrow s$ implies $\alpha\left(s_{n}\right) \rightarrow \alpha(s)$ including for sequences $s_{n} \rightarrow \infty$ as well as for any $s \in \mathbb{R}_{>0}$ such that $\alpha(s)=\infty$.

Definition 8 A function $\alpha: \overline{\mathbb{R}}_{\geq 0} \rightarrow \overline{\mathbb{R}}_{\geq 0}$ is said to be of class- $\overline{\mathcal{K}}$ if it is continuous, zero at zero, strictly increasing on the range $\mathbb{R}_{\geq 0}$ and infinite otherwise.

By strictly increasing on the range $\mathbb{R}_{\geq 0}$ we mean that on the set

$$
\left\{s \in \overline{\mathbb{R}}_{\geq 0}: \alpha(s) \neq \infty\right\}
$$

the function $\alpha$ is strictly increasing. An example of a function $\alpha \in \overline{\mathcal{K}}$ is

$$
\alpha(s)= \begin{cases}\arctan (s), & s \in \mathbb{R}_{\geq 0} \\ \frac{\pi}{2}, & s=\infty\end{cases}
$$

Another example of a function $\varphi \in \overline{\mathcal{K}}$ is

$$
\varphi(s)= \begin{cases}\tan (s), & s \in\left[0, \frac{\pi}{2}\right) \\ \infty, & s \in\left[\frac{\pi}{2}, \infty\right]\end{cases}
$$

Ito defined the pseudo-inverse of a function $\alpha \in \overline{\mathcal{K}}$ as

$$
\alpha^{\ominus}(s) \doteq \begin{cases}\alpha^{-1}(s), & s \in\left[0, \lim _{\tau \rightarrow \infty} \alpha(\tau)\right) \\ \sup \left\{s \in \mathbb{R}_{\geq 0}: \alpha(s)<\infty\right\}, & \text { otherwise. }\end{cases}
$$

We note that, with these definitions, if a function $\alpha \in \overline{\mathcal{K}}$ then $\alpha^{\ominus} \in \overline{\mathcal{K}}$. Furthermore, we may state the following result.

Lemma 30 Let $\alpha \in \overline{\mathcal{K}}$. If

$$
\sup \left\{s \in \mathbb{R}_{\geq 0}: \alpha(s)<\infty\right\}=\infty
$$

then, for all $s \in \overline{\mathbb{R}}_{\geq 0}$,

$$
\alpha^{\ominus} \circ \alpha(s)=s \text { and } \alpha \circ \alpha^{\ominus}(s) \leq s \text {. }
$$

On the other hand, if

$$
\sup \left\{s \in \mathbb{R}_{\geq 0}: \alpha(s)<\infty\right\}=c<\infty
$$

then, for all $s \in \overline{\mathbb{R}}_{\geq 0}$,

$$
\alpha^{\ominus} \circ \alpha(s) \leq s \text { and } \alpha \circ \alpha^{\ominus}(s)=s \text {. }
$$


Proof Let $a=\lim _{\tau \rightarrow \infty} \alpha(\tau)$ so that $\alpha: \overline{\mathbb{R}}_{\geq 0} \rightarrow[0, a]$ and $\alpha^{-1}:[0, a) \rightarrow[0, \infty)$. If (24) holds then

$$
\alpha^{\ominus}(s)= \begin{cases}\alpha^{-1}(s), & s \in[0, a) \\ \infty, & s \geq a\end{cases}
$$

so that

$$
\alpha^{\ominus} \circ \alpha(s)=\left\{\begin{array}{ll}
s, & s \in[0, \infty) \\
\infty, & s=\infty
\end{array} \quad \text { and } \alpha \circ \alpha^{\ominus}(s)= \begin{cases}s, & s \in[0, a) \\
a, & s \geq a\end{cases}\right.
$$

Therefore, (25) holds.

If (26) holds then $\lim _{s \nearrow c} \alpha(s)=\infty$ (i.e., $\alpha(c)=\infty$ ) and

$$
\alpha^{\ominus}(s)= \begin{cases}\alpha^{-1}(s), & s \in[0, \infty) \\ c, & s=\infty\end{cases}
$$

so that

$$
\alpha^{\ominus} \circ \alpha(s)=\left\{\begin{array}{ll}
s, & s \in[0, c) \\
c, & s \geq c
\end{array} \quad \text { and } \alpha \circ \alpha^{\ominus}(s)= \begin{cases}s, & s \in[0, \infty) \\
\infty, & s=\infty\end{cases}\right.
$$

Therefore, (27) holds.

We observe that $\alpha, \varphi \in \overline{\mathcal{K}}$ from (21) and (22) are pseudo-inverses of each other; i.e., $\alpha^{\ominus}=\varphi$ and $\varphi^{\ominus}=\alpha$. Furthermore, $\alpha \in \overline{\mathcal{K}}$ satisfies (24) while $\varphi \in \overline{\mathcal{K}}$ satisfies (26).

Definition 9 A function $\sigma: \overline{\mathbb{R}}_{\geq 0} \rightarrow \overline{\mathbb{R}}_{\geq 0}$ is said to be of class- $\overline{\mathcal{L}}$ if it is continuous, strictly decreasing, and $\sigma(\infty)=0$.

For $\sigma: \overline{\mathbb{R}}_{\geq 0} \rightarrow \overline{\mathbb{R}}_{\geq 0}$, it is understood that strictly decreasing allows $\sigma(0)=\infty$ but requires $\sigma(s)<\infty$ for all $s \in \overline{\mathbb{R}}_{>0}$.

We define the pseudo-inverse of a function $\sigma \in \overline{\mathcal{L}}$ as

$$
\sigma^{\dagger}(s) \doteq \begin{cases}\infty, & s=0 \\ \sigma^{-1}(s), & s \in(0, \sigma(0)) \\ 0, & s \in[\sigma(0), \infty]\end{cases}
$$

Similar to the fact that the inverse of a function $\sigma \in \mathcal{L}$ is nearly of class- $\mathcal{L}$ (see Section 2), the pseudo-inverse of a function $\sigma \in \overline{\mathcal{L}}$ is nearly of class- $\overline{\mathcal{L}}$; i.e., $\sigma^{\dagger}$ : $\overline{\mathbb{R}}_{\geq 0} \rightarrow \overline{\mathbb{R}}_{\geq 0}$ is continuous, strictly decreasing for $s \in[0, \sigma(0))$, and $\sigma^{\dagger}(\infty)=0$.

We observe that, for all $s \in \overline{\mathbb{R}}_{\geq 0}$,

$$
\sigma^{\dagger} \circ \sigma(s)=s \text { and } \sigma \circ \sigma^{\dagger}(s) \leq s
$$


Constraining $\sigma \in \overline{\mathcal{L}}$ so that $\sigma(0)=\infty$ yields that $\sigma^{\dagger} \in \overline{\mathcal{L}}$ is a true inverse of $\sigma \in \overline{\mathcal{L}}$. For example, the function

$$
\sigma(s)= \begin{cases}\infty, & s=0 \\ \frac{1}{s}, & s \in(0, \infty) \\ 0, & s=\infty\end{cases}
$$

is of class- $\overline{\mathcal{L}}$ and is its own pseudo-inverse.

\subsection{Functions of class- $\mathcal{G}$}

The function class- $\mathcal{G}$ was defined in [40] by enlarging the class- $\mathcal{K}$ to include functions that are not strictly increasing. ${ }^{8}$

Definition 10 A function $\alpha: \mathbb{R}_{\geq 0} \rightarrow \mathbb{R}_{\geq 0}$ is said to be of class- $\mathcal{G}$ if it is continuous, nondecreasing, and $\alpha(0)=0$.

It is clear that any class- $\mathcal{G}$ function can be bounded from above by a function of class- $\mathcal{K}$. However, it is not the case that every class- $\mathcal{G}$ function can be bounded from below by a function of class- $\mathcal{K}$. For example, a function which is identically zero for all $s \in[0,1]$ and equal to $s-1$ for all $s>1$ is of class- $\mathcal{G}$, but cannot be bounded from below by a function of class- $\mathcal{K}$.

Since class- $\mathcal{G}$ functions are not strictly increasing, they may fail to be invertible. This shortcoming can typically be circumvented by allowing set-valued inverses or by the introduction of class- $\mathcal{K}$ upper and lower bounds, where especial care is required to properly deal with any lower bound.

An alternative approach is to make use of the extended real-valued functions defined in the previous section to construct approximate inverses. Define class- $\overline{\mathcal{G}}$ as the enlargement of class $-\overline{\mathcal{K}}$ to include functions that are not strictly increasing.

Lemma 31 [15, Proposition 1] Suppose $\gamma \in \overline{\mathcal{G}}$ is positive definite and $\alpha \in \overline{\mathcal{K}}$. Let $c \in \overline{\mathbb{R}}_{\geq 0}$ be given by $c=\sup \left\{s \in \overline{\mathbb{R}}_{\geq 0}: \alpha(s)<\infty\right\}$ and assume

$$
c<\infty \Rightarrow \gamma(\infty)<c
$$

Then, for all $s \in \overline{\mathbb{R}}_{\geq 0}$,

$$
\alpha \circ \gamma(s) \leq s \text { if and only if } \gamma \circ \alpha(s) \leq s
$$

The proof of the above lemma is provided in Appendix A.11.

\footnotetext{
8 These functions were introduced in [39] under the nomenclature of "gain functions", leading to the natural function class name of $\mathcal{G}$ in [40]. In [7], these functions were defined as class- $\overline{\mathcal{K}}$ while in [15] such functions were denoted by class- $\mathcal{J}$.
} 
As an example, suppose we wish to derive an approximate inverse for the positive definite function $\gamma \in \overline{\mathcal{G}}$ defined by

$$
\gamma(s)=\operatorname{sat}(2 s) \doteq\left\{\begin{array}{ll}
2 s, & s \in\left[0, \frac{1}{2}\right] \\
1, & s>\frac{1}{2}
\end{array} .\right.
$$

A well-defined inverse exists on $[0,1)$ but we encounter an obvious difficulty at the value of one. One possible approximation of an inverse for $\gamma$ is given by $\alpha \in \overline{\mathcal{K}}$ defined as

$$
\alpha(s) \doteq \begin{cases}\frac{1}{2} s, & s \in[0,1] \\ \frac{a-1}{2(a-s)}, & s \in[1, a) \\ \infty, & s \geq a\end{cases}
$$

for some $a>1$. We see that this extended real-valued function is the expected inverse on $[0,1]$ and goes to infinity arbitrarily close to one. It is straightforward to see that, for all $s \in \overline{\mathbb{R}}_{\geq 0}$, both $\alpha \circ \gamma(s) \leq s$ and $\gamma \circ \alpha(s) \leq s$, indicating that Lemma 31 captures a property of reasonable approximations of inverses of class $-\overline{\mathcal{G}}$ functions.

\subsection{Functions of class- $\mathcal{N}$}

The nomenclature of class- $\mathcal{N}$ has been used to denote two different function classes ${ }^{9}$. We here adopt the definition in [3].

Definition 11 A function $v: \mathbb{R} \rightarrow \mathbb{R}$ is said to be of class- $\mathcal{N}$ if it is continuous, nondecreasing, and unbounded below (that is, inf $v=-\infty$ ).

The following lemma is the key element in the proofs of Lemma 11 and Lemma 19.

Lemma 32 [3, Proposition IV.4] Suppose $\phi: \mathbb{R}^{2} \rightarrow \mathbb{R}$ is class- $\mathcal{N}$ in each of its arguments; i.e., $\phi(\cdot, y) \in \mathcal{N}$ for each fixed $y \in \mathbb{R}$ and $\phi(x, \cdot) \in \mathcal{N}$ for each fixed $x \in \mathbb{R}$. Then there exists $\kappa \in \mathcal{N}$ such that

$$
\phi(x, y) \leq \kappa(x)+\kappa(y), \quad \forall(x, y) \in \mathbb{R}^{2} .
$$

To prove Lemma 11 , given $\alpha: \mathbb{R}_{\geq 0}^{2} \rightarrow \mathbb{R}_{\geq 0}$ of class- $\mathcal{K}$ in each of its arguments independently, the function

$$
\phi(x, y) \doteq \log \left(\alpha\left(e^{x}, e^{y}\right)\right), \quad \forall x, y \in \mathbb{R}
$$

where $\log$ denotes the natural logarithm, can be shown to be of class- $\mathcal{N}$. Applying Lemma 32, Lemma 1, and taking an exponential on both sides of the resulting inequality gives the desired result with $\hat{\alpha} \in \mathcal{K}$ bounding $\varphi(s) \doteq e^{k(\log (s))}$ from above.

\footnotetext{
9 In [2], the function class- $\mathcal{N}$ denotes those functions which map $\mathbb{R}_{\geq 0}$ to $\mathbb{R}_{\geq 0}$ and are continuous and increasing. In this case, class- $\mathcal{K}$ is the subset of class- $\mathcal{N}$ functions that are also zero at zero.
} 
The core idea of the proof of Lemma 19 similarly requires defining an appropriate function $\phi$. Define $|x|_{+} \doteq \max \{0, x\}$ and

$$
\phi(x, y) \doteq-\log \left(\tilde{\beta}\left(\frac{1}{|x|_{+}},|y|_{+}\right)\right)-|-x|_{+}-|-y|_{+}, \quad \forall x, y \in \mathbb{R},
$$

for a function $\tilde{\beta} \in \mathcal{K} \mathcal{L}$ that is a lower bound for the given $\beta \in \mathcal{K} \mathcal{L}$ with certain desirable properties. Again we apply Lemma 32, Lemma 1, and take exponentials on both sides of the resulting inequality. Some straightforward manipulations then yield the desired result.

\section{Monotone aggregation functions}

To compare two vectors $x, y \in \mathbb{R}_{>0}^{n}$, we use $x \gg y$ if and only if $x_{i}>y_{i}$ for all $i \in\{1, \ldots, n\}$. In other words, each element of $x \in \mathbb{R}_{\geq 0}^{n}$ must be greater than the corresponding element in $y \in \mathbb{R}_{\geq 0}^{n}$.

A generalization of class- $\mathcal{K}$ functions allowing a domain of $\mathbb{R}_{\geq 0}^{n}$, rather than a domain of $\mathbb{R}_{\geq 0}$, was introduced in [33] (see also [6]). These so-called monotone aggregation functions are defined as follows:

Definition 12 A continuous function $\mu: \mathbb{R}_{\geq 0}^{n} \rightarrow \mathbb{R}_{\geq 0}$ is a monotone aggregation function $\left(M A F_{n}\right)$ if it is positive definite and strictly monotone; i.e., for any $x, y \in \mathbb{R}_{\geq 0}^{n}$, if $x \gg y$ then $\mu(x)>\mu(y)$. The class of unbounded monotone aggregation functions on $\mathbb{R}_{\geq 0}^{n}$ is denoted by $\mathrm{MAF}_{n, \infty}$.

Together with gain matrices describing the interconnection structure of large-scale systems, monotone aggregation functions were used in [33] and subsequent works to provide results on stability of large-scale systems, particularly through the use of small-gain theorems.

Examples of monotone aggregation functions include maximization and all $p$ norms on $\mathbb{R}^{n}$. As such, they provide a general and elegant formulation encapsulating and expanding the standard input-to-state stability estimates for systems with inputs. In particular, for $\dot{x}=f(x, w)$, we can subsume both the estimate

$$
|x(t)| \leq \beta(|x(0)|, t)+\gamma\left(\|w\|_{\infty}\right)
$$

and the estimate

$$
|x(t)| \leq \max \left\{\beta(|x(0)|, t), \gamma\left(|| w \|_{\infty}\right)\right\}
$$

by the use of a function $\mu \in \mathrm{MAF}_{2}$ and the estimate

$$
|x(t)| \leq \mu\left(\beta(|x(0)|, t), \gamma\left(\|w\|_{\infty}\right)\right)
$$

There are several useful and straightforward relationships between functions of class- $\mathcal{K}$ and monotone aggregation functions: 
- The function classes $\mathcal{K}$ and $\mathrm{MAF}_{1}$ are equivalent.

- If $\alpha \in \mathcal{K}$ and $\mu \in \mathrm{MAF}_{n}$, then $\alpha \circ \mu \in \mathrm{MAF}_{n}$.

- If $\alpha_{i} \in \mathcal{K}$ for $i=1, \ldots, n$, and $\mu \in \mathrm{MAF}_{n}$, then the function

$$
\mu_{\alpha}\left(s_{1}, \ldots, s_{n}\right) \doteq \mu\left(\alpha_{1}\left(s_{1}\right), \ldots, \alpha_{n}\left(s_{n}\right)\right)
$$

is in $\mathrm{MAF}_{n}$.

- Denote the vector of all ones in $\mathbb{R}^{n}$ by $\mathbf{1}_{n}$. If $\mu \in \mathrm{MAF}_{n}$ then $\mu\left(s \mathbf{1}_{n}\right), s \in \mathbb{R}_{\geq 0}$, is a function of class- $\mathcal{K}$.

Despite the equivalence of class- $\mathcal{K}$ and $\mathrm{MAF}_{1}$, monotone aggregation functions are in fact different to functions $\alpha: \mathbb{R}_{>0}^{n} \rightarrow \mathbb{R}_{\geq 0}, n \in \mathbb{Z}_{>1}$, that are of class- $\mathcal{K}$ in each argument, or what might be termed jointly class- $\mathcal{K}$. For example, in the case of a jointly class- $\mathcal{K}$ function of two arguments, $\alpha \in \mathcal{K}^{2}$, we see that $\alpha(s, 0)=0$ for all $s \in \mathbb{R}_{\geq 0}$. On the other hand, a function $\mu \in \mathrm{MAF}_{2}$ is positive definite; i.e., $\mu(s, 0)>0$ for all $s \in \mathbb{R}_{>0}$.

Lemma 33 Given $\mu \in \mathrm{MAF}_{n}$ and integers $n_{1}, n_{2}$ such that $n_{1}+n_{2}=n$, there exist $\mu_{1} \in \mathrm{MAF}_{n_{1}}$ and $\mu_{2} \in \mathrm{MAF}_{n_{2}}$ such that

$$
\mu\left(s_{1}, \ldots, s_{n}\right) \leq \mu_{1}\left(s_{1}, \ldots, s_{n_{1}}\right)+\mu_{2}\left(s_{n_{1}+1}, \ldots, s_{n}\right)
$$

for all $\left(s_{1}, \ldots, s_{n}\right) \in \mathbb{R}_{\geq 0}^{n}$.

Proof Denote a ball of radius $r \geq 0$ in the positive orthant by $\mathcal{B}_{r}^{+}$and let $\alpha \in \mathcal{K}$ be given by

$$
\alpha(s) \doteq \max _{x \in \mathcal{B}_{s}^{+}} \mu(x)
$$

That $\alpha \in \mathcal{K}$ follows directly from the fact that $\mu \in \mathrm{MAF}_{n}$. We see that

$$
\begin{aligned}
\mu\left(s_{1}, \ldots, s_{n}\right) & \leq \alpha\left(s_{1}\right)+\cdots+\alpha\left(s_{n}\right) \\
& =\sum_{i=1}^{n_{1}} \alpha\left(s_{i}\right)+\sum_{i=n_{1}+1}^{n} \alpha\left(s_{i}\right) \\
& =: \mu_{1}\left(s_{1}, \ldots, s_{n_{1}}\right)+\mu_{2}\left(s_{n_{1}+1}, \ldots, s_{n}\right) .
\end{aligned}
$$

That $\mu_{1} \in \mathrm{MAF}_{n_{1}}$ and $\mu_{2} \in \mathrm{MAF}_{n_{2}}$ follow from the fact that the sum of $n$ functions of class- $\mathcal{K}$ is a function of class-MAF ${ }_{n}$.

The above result leads to the following result from [8] allowing a general monotone aggregation function to be bounded from above by a summation. We denote by $\mathcal{K}_{\infty} \cup$ $\{0\}$ those functions that are either class- $\mathcal{K}_{\infty}$ or identically zero.

Lemma 34 For any $\mu \in \operatorname{MAF}_{n+1}, \beta \in \mathcal{K} \mathcal{L}$, and $\alpha_{i} \in \mathcal{K}_{\infty} \cup\{0\}, i=1, \ldots, n$ there exist $\hat{\mu} \in \mathrm{MAF}_{n-1}, \hat{\beta} \in \mathcal{K} \mathcal{L}, \hat{\alpha}_{n} \in \mathcal{K}_{\infty} \cup\{0\}$ such that 
$\mu\left(\beta\left(s_{0}, t\right), \alpha_{1}\left(s_{1}\right), \ldots, \alpha_{n}\left(s_{n}\right)\right) \leq \hat{\beta}\left(s_{0}, t\right)+\hat{\mu}\left(\alpha_{1}\left(s_{1}\right), \ldots, \alpha_{n-1}\left(s_{n-1}\right)\right)+\hat{\alpha}_{n}\left(s_{n}\right)$

for all $t, s_{0}, \ldots, s_{n} \in \mathbb{R}_{\geq 0}$.

Proof We apply Lemma 33 twice to the function $\mu \in \mathrm{MAF}_{n+1}$ to obtain $\hat{\mu}_{1} \in \mathrm{MAF}_{1}$, $\hat{\mu}_{2} \in \mathrm{MAF}_{1}$, and $\hat{\mu} \in \mathrm{MAF}_{n-1}$. With the equivalence of class- $\mathcal{K}$ and $\mathrm{MAF}_{1}$, we can define $\hat{\beta} \doteq \hat{\mu}_{1} \circ \beta \in \mathcal{K} \mathcal{L}$ and $\hat{\alpha}_{n} \doteq \hat{\mu}_{2} \circ \alpha_{n} \in \mathcal{K}$.

Acknowledgments The author would like to thank Lars Grüne, Björn Rüffer, Andy Teel, and Fabian Wirth for comments on early drafts of this manuscript. The work of the anonymous reviewers is also gratefully acknowledged.

Open Access This article is distributed under the terms of the Creative Commons Attribution License which permits any use, distribution, and reproduction in any medium, provided the original author(s) and the source are credited.

\section{Appendix}

In this appendix, we present proofs of selected lemmas. The proofs generally follow those presented in the citation accompanying the associated lemma.

\section{A.1 Proof of Lemma 3 (Smooth approximations)}

Without loss of generality, assume $\varepsilon<\frac{4}{3} \sup \alpha$. Define $a \doteq \sup \alpha$ and

$$
I \doteq \sup \left\{i \in \mathbb{Z}_{>0}:(i+1) \frac{\varepsilon}{4} \leq a-\frac{\varepsilon}{4}\right\} \text {. }
$$

Note that if $\alpha \in \mathcal{K}_{\infty}$, then both $a$ and $I$ are taken to be infinity. If $\alpha \in \mathcal{K} \backslash \mathcal{K}_{\infty}$, then the supremum defining $I$ is a maximum. Finally, the fact that the set is nonempty follows from the constraint that $\varepsilon<\frac{4}{3} a$.

Define the double-sided infinite sequence

$$
R_{i} \doteq \begin{cases}\frac{\varepsilon}{4} 2^{i}, & i \in \mathbb{Z}_{\leq 0} \\ (i+1) \frac{\varepsilon}{4}, & i \in\{1, \ldots, I\} \\ \left.(I+1) \frac{\varepsilon}{4}+\left(a-(I+1) \frac{\varepsilon}{4}\right)\right) \sum_{j=1}^{i-I} 2^{-j}, & i \in \mathbb{Z}_{>I}\end{cases}
$$

where, from the definition of $I$ in (32), we have that $R_{I} \leq a-\frac{\varepsilon}{4}$. We observe that $R_{i}<R_{i+1}$ for all $i \in \mathbb{Z}, \lim _{i \rightarrow-\infty} R_{i}=0$ and $\lim _{i \rightarrow \infty} R_{i}=a$. Therefore, $R_{i}$ partitions the open interval $(0, a)$.

Note that, if $\alpha \in \mathcal{K}_{\infty}$, then $I=\infty$ and the partition definition (33) only requires the regions $i \in \mathbb{Z}_{\leq 0}$ and $i \in \mathbb{Z}_{>0}$.

Define a strictly increasing continuous function $\bar{\alpha}_{2}: \mathbb{R}_{\geq 0} \rightarrow \mathbb{R}_{\geq 0}$ by $\bar{\alpha}_{2}(0)=0$ and

$$
\bar{\alpha}_{2}(s) \doteq R_{i+1}+\left(s-\alpha^{-1}\left(R_{i-1}\right)\right) \frac{R_{i+2}-R_{i+1}}{\alpha^{-1}\left(R_{i}\right)-\alpha^{-1}\left(R_{i-1}\right)},
$$


for all $s \in\left[\alpha^{-1}\left(R_{i-1}\right), \alpha^{-1}\left(R_{i}\right)\right)$. That $\bar{\alpha}_{2}$ is continuous at the origin follows from the fact that $i \rightarrow-\infty$ when $s \rightarrow 0$ and both $\lim _{i \rightarrow-\infty} R_{i}=0$ and $\lim _{i \rightarrow-\infty}\left(R_{i+2}-\right.$ $\left.R_{i+1}\right)=0$.

We see that, for all $s \in\left[\alpha^{-1}\left(R_{i-1}\right), \alpha^{-1}\left(R_{i}\right)\right)$,

$$
\alpha(s)+\left(R_{i+1}-R_{i}\right)<\bar{\alpha}_{2}(s)<\alpha(s)+\left(R_{i+2}-R_{i-1}\right) .
$$

It is straightforward to calculate

$$
R_{i+1}-R_{i}= \begin{cases}\frac{\varepsilon}{4} 2^{i}, & i \in \mathbb{Z}_{\leq 0} \\ \frac{\varepsilon}{4}, & i \in\{1, \ldots, I-1\} \\ \frac{\varepsilon}{4} 2^{-(i+1-I)}, & i \in \mathbb{Z}_{\geq I}\end{cases}
$$

A standard regularization technique applied to $\bar{\alpha}_{2}$ then yields a function $\alpha_{2} \in \mathcal{K}$ that is smooth on $\mathbb{R}_{>0}$ and satisfies

$$
\alpha(s) \leq \alpha_{2}(s)<\alpha(s)+\varepsilon, \quad \forall s \in \mathbb{R}_{\geq 0}
$$

Finally, the smooth function $\alpha_{1} \in \mathcal{K}$ can be obtained by a similar procedure where a strictly increasing continuous function similar to (34) is defined by

$$
\bar{\alpha}_{1}(s) \doteq R_{i-1}+\left(s-\alpha^{-1}\left(R_{i-1}\right)\right) \frac{R_{i}-R_{i-1}}{\alpha^{-1}\left(R_{i}\right)-\alpha^{-1}\left(R_{i-1}\right)},
$$

for all $s \in\left[\alpha^{-1}\left(R_{i-1}\right), \alpha^{-1}\left(R_{i}\right)\right)$ and $\bar{\alpha}_{1}(0)=0$.

Remark 1 The proof of Lemma 4 follows a similar approach with the only significant difference being the definition of the partition. This is done by first defining

$$
I \doteq \min \left\{i \in \mathbb{Z}_{<0}:(-i+1) \frac{\varepsilon}{4} \leq \sigma(0)-\frac{\varepsilon}{4}\right\}
$$

(compare with (32)) and then

$$
R_{i} \doteq \begin{cases}\frac{\varepsilon}{4} 2^{-i}, & i \in \mathbb{Z}_{\geq 0} \\ (-i+1) \frac{\varepsilon}{4}, & i \in\{I, \ldots,-1\} \\ R_{I}+\left(\sigma(0)-R_{I}\right) \sum_{j=1}^{-i+I} 2^{-j}, & i \in \mathbb{Z}_{<I}\end{cases}
$$

(compare with (33)). The proof then follows that of Lemma 3, mutatis mutandis, by defining similar continuous, though strictly decreasing, functions.

\section{A.2 Proof of Lemma 7 (Sontag's Lemma on $\mathcal{K} \mathcal{L}$-Estimates)}

We require the following: 
Claim Given $\beta \in \mathcal{K} \mathcal{L}$, there exist $\alpha \in \mathcal{K}_{\infty}$ and $\sigma \in \mathcal{L}$, smooth on $\mathbb{R}_{>0}$ such that

$$
\beta(\alpha(t), t) \leq \sigma(t), \quad \forall t \in \mathbb{R}_{\geq 0}
$$

Proof To see this, let $\left\{\varepsilon_{k}\right\}_{k=1}^{\infty}$ be a sequence strictly decreasing to zero. Since $\beta \in \mathcal{K} \mathcal{L}$, there exists a strictly increasing sequence $\left\{t_{k}\right\}_{k=1}^{\infty}$ satisfying $\lim _{k \rightarrow \infty} t_{k}=\infty$ such that

$$
\beta\left(k+1, t_{k}\right) \leq \varepsilon_{k}, \quad \forall k \in \mathbb{Z}_{\geq 1}
$$

Let $t_{0}=0$ and $\varepsilon_{0}=\max \left\{\beta(1,0), 2 \varepsilon_{1}\right\}$.

Now, let $\alpha: \mathbb{R}_{\geq 0} \rightarrow \mathbb{R}_{\geq 0}$ be any class- $\mathcal{K}_{\infty}$ function bounded from above by the nondecreasing piecewise constant function defined by $\hat{\alpha}(t)=k+1$ for $t \in\left[t_{k}, t_{k+1}\right)$. Additionally, let $\sigma: \mathbb{R}_{\geq 0} \rightarrow \mathbb{R}_{\geq 0}$ be any smooth on $\mathbb{R}_{>0}$ class- $\mathcal{L}$ function that is bounded from below by the nonincreasing piecewise constant function defined by $\hat{\sigma}(t)=\varepsilon_{k}$ for $t \in\left[t_{k}, t_{k+1}\right.$ ). Then, for every $k \in \mathbb{Z}_{\geq 0}$ and $t \in\left[t_{k}, t_{k+1}\right.$ ) (and hence for all $t \in \mathbb{R}_{\geq 0}$ )

$$
\beta(\alpha(t), t) \leq \beta(\hat{\alpha}(t), t) \leq \beta(k+1, t) \leq \beta\left(k+1, t_{k}\right) \leq \varepsilon_{k} \leq \sigma(t) .
$$

Given $\beta \in \mathcal{K} \mathcal{L}$, take $\alpha \in \mathcal{K}_{\infty}$ and $\sigma \in \mathcal{L}$ from the above claim. Since $\sigma \in$ $\mathcal{L}$ and smooth on $\mathbb{R}_{>0}, \sigma^{-1}:(0, \sigma(0)] \rightarrow \mathbb{R}_{\geq 0}$ is continuous, smooth on $\mathbb{R}_{>0}$, strictly decreasing, and satisfies $\lim _{s \rightarrow 0} \sigma^{-1}(s)=+\infty$. Consequently, the function $e^{-2 \lambda \sigma^{-1}(s)}$ is continuous, smooth on $\mathbb{R}_{>0}$, and strictly increasing for $s \in(0, \sigma(0)]$. Let $\pi: \mathbb{R}_{\geq 0} \rightarrow \mathbb{R}_{\geq 0}$ be a continuous, smooth on $\mathbb{R}_{>0}$, positive, nondecreasing function that satisfies $\pi(0)=0$,

$$
\pi(s)=\frac{1}{2 \sigma(0)} e^{-2 \lambda \sigma^{-1}(s)}, \quad s \in(0, \sigma(0)]
$$

and $\pi(s)<\frac{1}{\sigma(0)}$ for $s \geq \sigma(0)$.

We now define $\alpha_{1}(s) \doteq \int_{0}^{s} \pi(\tau) d \tau$. It is clear that $\alpha_{1} \in \mathcal{K}_{\infty}$. Clearly $\alpha_{1}(\cdot)$ is globally Lipschitz on $\mathbb{R}_{\geq 0}$ and smooth on $\mathbb{R}_{>0}$. Furthermore, since $\pi(\cdot)$ is nondecreasing

$$
\alpha_{1}(s)=\int_{0}^{s} \pi(\tau) d \tau \leq s \pi(s)=s \alpha_{1}^{\prime}(s), \quad \forall s \in \mathbb{R}_{>0}
$$

and, for $s \in(0, \sigma(0)]$

$$
\alpha_{1}(s) \leq s \pi(s) \leq \sigma(0) \pi(s)=\frac{1}{2} e^{-2 \lambda \sigma^{-1}(s)} .
$$

This property in combination with (38) allows us to write

$$
\alpha_{1}(\beta(\alpha(t), t)) e^{2 \lambda t} \leq \alpha_{1}(\sigma(t)) e^{2 \lambda t} \leq \frac{1}{2} e^{-2 \lambda t} e^{2 \lambda t}<1 .
$$


To derive the desired expression for $\alpha_{2} \in \mathcal{K}_{\infty}$, we consider the argument $s \in \mathbb{R}_{\geq 0}$ on two regions and calculate bounds on $\alpha_{1}(\beta(s, t)) e^{\lambda t}$. First, for $s \in(0, \alpha(t)]$ we see that

$$
\begin{aligned}
\alpha_{1}(\beta(s, t)) e^{\lambda t} & =\sqrt{\alpha_{1}(\beta(s, 0))} \sqrt{\frac{\alpha_{1}(\beta(s, t))}{\alpha_{1}(\beta(s, 0))}} \sqrt{\alpha_{1}(\beta(s, t)) e^{2 \lambda t}} \\
& \leq \sqrt{\alpha_{1}(\beta(s, 0))} \sqrt{\alpha_{1}(\beta(\alpha(t), t)) e^{2 \lambda t}} \\
& \leq \sqrt{\alpha_{1}(\beta(s, 0))},
\end{aligned}
$$

where the first inequality follows from the fractional term being less than one and $s \leq \alpha(t)$, while the second inequality follows from (40).

Second, for $s \geq \alpha(t)$ we see that

$$
\alpha_{1}(\beta(s, t)) e^{\lambda t} \leq \alpha_{1}(\beta(s, 0)) e^{\lambda \alpha^{-1}(s)} .
$$

We observe that both upper bounds (41) and (42) are of class- $\mathcal{K}_{\infty}$ and note that the maximum of two $\mathcal{K}_{\infty}$ functions is itself a $\mathcal{K}_{\infty}$ function. Hence, setting

$$
\alpha_{2}(s) \doteq \max \left\{\sqrt{\alpha_{1}(\beta(s, 0))}, \alpha_{1}(\beta(s, 0)) e^{\lambda \alpha^{-1}(s)}\right\}
$$

completes the proof.

\section{A.3 Proof of Lemma 10 (Weak triangle inequality)}

We here prove the weak triangle inequality in the form of Eq. (10): given $\alpha \in \mathcal{K}$, for any $\varphi \in \mathcal{K}_{\infty}$

$$
\alpha(a+b) \leq \alpha \circ(\varphi+\mathrm{id})(a)+\alpha \circ\left(\varphi^{-1}+\mathrm{id}\right)(b), \quad \forall a, b, \in \mathbb{R}_{\geq 0} .
$$

We consider two cases: $\varphi(a) \geq b$ and $\varphi(a)<b$. In the first case, making use of the invertibility of $\mathcal{K}_{\infty}$ functions, we have that $a \geq \varphi^{-1}(b)$ so that

$$
\begin{aligned}
\alpha(\varphi(a)+a)+\alpha\left(\varphi^{-1}(b)+b\right) & \geq \alpha\left(\varphi \circ \varphi^{-1}(b)+a\right)+\alpha\left(\varphi^{-1}(b)+b\right) \\
& \geq \alpha(b+a)+\alpha\left(\varphi^{-1}(b)+b\right) \geq \alpha(a+b) .
\end{aligned}
$$

On the other hand, if $\varphi(a)<b$, then $a<\varphi^{-1}(b)$ and we see that

$$
\begin{aligned}
\alpha(\varphi(a)+a)+\alpha\left(\varphi^{-1}(b)+b\right) & >\alpha(\varphi(a)+a)+\alpha(a+b) \\
& \geq \alpha(a+b)
\end{aligned}
$$

Combining (43) and (44) then yields the result. 


\section{A.4 Proof of Lemma 14 (Class- $\mathcal{L}$ upper bound)}

Using the fact that a class- $\mathcal{K}$ function of a class- $\mathcal{L}$ is again a class- $\mathcal{L}$ function, we see that if $\sigma \in \mathcal{L}$ then $\sqrt{\sigma} \in \mathcal{L}$. Furthermore, since $\sigma \in \mathcal{L}$ is strictly decreasing, we know that $\sigma(t+\tau) \leq \sigma(t)$ for all $t, \tau \in \mathbb{R}_{\geq 0}$. Let $\sigma_{i} \in \mathcal{L}$ satisfy $\sigma_{i}(t) \geq \sqrt{\sigma(t)}$ for $i=1,2$ and all $t \in \mathbb{R}_{\geq 0}$. Then, for all $r, s \in \mathbb{R}_{\geq 0}$ we may write

$$
\sigma_{1}(r) \sigma_{2}(s) \geq \sqrt{\sigma(r)} \sqrt{\sigma(s)} \geq \sqrt{\sigma(r+s)} \sqrt{\sigma(r+s)}=\sigma(r+s)
$$

\section{A.5 Proof of Lemma 18 (Positive definite functions)}

Without loss of generality we assume $\rho(x) \rightarrow 0$ as $|x| \rightarrow \infty$. (If this is not the case, since we are interested in deriving a lower bound for $\rho(x)$, we can instead consider any positive definite function $\hat{\rho}: \mathbb{R}^{n} \rightarrow \mathbb{R}_{\geq 0}$ satisfying $\hat{\rho}(x) \leq \rho(x)$ for all $x \in \mathbb{R}^{n}$ and $\hat{\rho}(x) \rightarrow 0$ as $|x| \rightarrow \infty$.) With this assumption, and the facts that $\rho(0)=0$ and $\rho(x)>0$ for all $x \in \mathbb{R}^{n} \backslash\{0\}$, we know that the function has a global maximum, $x^{*} \in \mathbb{R}^{n}$ and that $\rho\left(x^{*}\right)>0$.

We define the continuous, positive, and nondecreasing function

$$
\tilde{\alpha}(s) \doteq \begin{cases}\min _{s \leq|y| \leq\left|x^{*}\right|} \frac{\rho(y)}{\rho\left(x^{*}\right)}, & s \leq\left|x^{*}\right|, \\ \min _{|y|=\left|x^{*}\right|} \frac{\rho(y)}{\rho\left(x^{*}\right)}, & s>\left|x^{*}\right| .\end{cases}
$$

We also define the continuous, positive, and nonincreasing function

$$
\tilde{\sigma}(s) \doteq \begin{cases}\min _{|y|=\left|x^{*}\right|} \frac{\rho(y)}{\rho\left(x^{*}\right)}, & s<\left|x^{*}\right|, \\ \min _{\left.\left|x^{*}\right| \leq|y| \leq s\right\}} \frac{\rho(y)}{\rho\left(x^{*}\right)}, & s \geq\left|x^{*}\right| .\end{cases}
$$

Since $\min _{|y|=\left|x^{*}\right|} \frac{\rho(y)}{\rho\left(x^{*}\right)} \leq 1$, we observe that the product of these two functions satisfies

$$
\tilde{\alpha}(|x|) \tilde{\sigma}(|x|) \leq \frac{\rho(x)}{\rho\left(x^{*}\right)} .
$$

To complete the proof, we define $\alpha \in \mathcal{K}_{\infty}$ and $\sigma \in \mathcal{L}$ by

$$
\begin{aligned}
\alpha(s) & \doteq \rho\left(x^{*}\right) \tilde{\alpha}(s) s \\
\sigma(s) & \doteq \tilde{\sigma}(s) \frac{1}{1+s} .
\end{aligned}
$$


We then see that, with (45),

$$
\alpha(|x|) \sigma(|x|)=\rho\left(x^{*}\right) \tilde{\alpha}(|x|) \tilde{\sigma}(|x|) \frac{|x|}{1+|x|} \leq \rho(x) .
$$

\section{A.6 Proof of Lemma 20 (Comparison principle)}

We first demonstrate the result in the case of $\lambda=1$. The general result will then follow from a standard rescaling of time. Given the continuous, positive definite function $\rho: \mathbb{R}_{\geq 0} \rightarrow \mathbb{R}_{\geq 0}$ of the lemma, we define

$$
\hat{\rho}(s) \doteq \min \{s, \rho(s)\}
$$

and note that an absolutely continuous function $y:[0, T] \rightarrow \mathbb{R}_{\geq 0}$ which satisfies (15) must also satisfy

$$
\dot{y}(t) \leq-\hat{\rho}(y(t)), \quad \text { for almost all } t \in[0, T] .
$$

For $s \in(0, \infty)$ define

$$
\eta(s) \doteq-\int_{1}^{s} \frac{\mathrm{d} \tau}{\hat{\rho}(\tau)}
$$

We observe that $\eta(s)$ is continuously differentiable and strictly decreasing for $s \in$ $(0, \infty)$. Additionally,

$$
\lim _{s \rightarrow 0^{+}} \eta(s) \geq \lim _{s \rightarrow 0^{+}} \int_{s}^{1} \frac{\mathrm{d} \tau}{\tau}=+\infty
$$

and with

$$
0<c \doteq-\lim _{s \rightarrow \infty} \eta(s)
$$

(where it is possible that $c=+\infty)$ we see that $\eta:(0, \infty) \rightarrow(-c, \infty)$ and $\eta^{-1}$ : $(-c, \infty) \rightarrow(0, \infty)$. We further note that $\eta^{-1}$ is continuous and strictly decreasing to zero. We can then define

$$
\beta(s, t) \doteq \begin{cases}0, & s=0 \\ \eta^{-1}(\eta(s)+t), & s>0\end{cases}
$$

for all $s, t \in \mathbb{R}_{\geq 0}$. 
Since $\eta^{-1}(\cdot)$ is strictly decreasing to zero and continuous, $\beta(s, \cdot) \in \mathcal{L}$ for all $s \in \mathbb{R}_{\geq 0}$. To see that $\beta(s, t)$ is continuous at $s=0$ for each fixed $t \in \mathbb{R}_{\geq 0}$, fix $\varepsilon>0$. Since $\eta^{-1}(\cdot)$ is continuous and strictly decreasing to zero, there exists $N(\varepsilon)>0$ so that if $\eta(s)>N(\varepsilon)$ then $\eta^{-1}(\eta(s)+t)<\varepsilon$ for all $t \in \mathbb{R}_{\geq 0}$. Furthermore, since $\eta(\cdot)$ is continuous and satisfies (48), there exists $\delta_{N(\varepsilon)}>0$ so that if $s<\delta_{N(\varepsilon)}$ then $\eta(s)>N(\varepsilon)$ and therefore $\beta(s, t)<\varepsilon$. Finally, since both $\eta(\cdot)$ and $\eta^{-1}(\cdot)$ are strictly decreasing and continuous, $\beta(\cdot, t) \in \mathcal{K}$ for all $t \in \mathbb{R}_{\geq 0}$. Therefore, $\beta \in \mathcal{K} \mathcal{L}$.

We note that (46) and the condition that $y(t) \geq 0$ for all $t \in[0, T]$ imply that if there exists a $\bar{t} \in[0, T]$ such that $y(\bar{t})=0$, then $y(t) \equiv 0$ for all $t \in[\bar{t}, T]$. In the event that such a time exists, let $t^{*} \doteq \min \{t \in[0, T]: y(t)=0\}$. We then see that, for all $t \in\left[t^{*}, T\right]$, the bound $y(t) \leq \beta\left(y_{0}, t\right)$ is trivially satisfied. Note that if $y_{0}=0$, then $t^{*}=0$ and we are done. Hence, we subsequently assume $y_{0}>0$.

For $t \in\left[0, t^{*}\right)$ (or $t \in[0, T]$ if $t^{*}$ fails to exist), we observe that $\hat{\rho}(y(t))>0$ so that (46) implies

$$
\frac{\dot{y}(t)}{\hat{\rho}(y(t))} \leq-1 \Rightarrow \int_{0}^{t} \frac{\mathrm{d}(y(\tau))}{\hat{\rho}(y(\tau))} \leq-t
$$

and the change of variables $y(\tau)=r$ yields

$$
\int_{y(t)}^{y_{0}} \frac{\mathrm{d} r}{\hat{\rho}(r)} \geq t \Rightarrow-\int_{1}^{y(t)} \frac{\mathrm{d} r}{\hat{\rho}(r)}+\int_{1}^{y_{0}} \frac{\mathrm{d} r}{\hat{\rho}(r)} \geq t .
$$

With the definition (47) we see that this implies

$$
\eta(y(t)) \geq \eta\left(y_{0}\right)+t, \quad \forall t \in\left[0, t^{*}\right) .
$$

Since $\eta^{-1}$ is strictly decreasing, and since the bound is trivially satisfied for $t \in\left[t^{*}, T\right]$, we have

$$
y(t) \leq \eta^{-1}\left(\eta\left(y_{0}\right)+t\right)=\beta\left(y_{0}, t\right), \quad \forall t \in[0, T]
$$

as required.

Finally, by taking the time rescaling $\tau=\lambda t$, we see that $\dot{y}(t) \leq-\lambda \hat{\rho}(y(t))$ for almost all $t \in[0, T]$ becomes

$$
\frac{\mathrm{d}}{\mathrm{d} \tau} y(\tau / \lambda) \leq-\hat{\rho}(y(\tau / \lambda)), \quad \text { for almost all } \tau \in[0, \lambda T]
$$

Following the above, we have a function $\beta \in \mathcal{K} \mathcal{L}$ such that

$$
y(\tau / \lambda) \leq \beta\left(y_{0}, \tau\right), \quad \forall \tau \in[0, \lambda T]
$$


and hence

$$
y(t) \leq \beta\left(y_{0}, \lambda t\right), \quad \forall t \in[0, T] .
$$

\section{A.7 Proof of Lemma 23 (A factorization of Class- $\mathcal{K}$ functions)}

Let $\varphi_{1}, \varphi_{2} \in \mathcal{K}_{\infty}$ be such that $\varphi_{1}+\varphi_{2}=\mathrm{id}$. We observe that for $\alpha \in \mathcal{K}$, (id $+\varphi_{2} \circ \alpha$ ) is of class- $\mathcal{K}_{\infty}$ and, as such, (id $\left.+\varphi_{2} \circ \alpha\right)^{-1}$ exists and is of class- $\mathcal{K}_{\infty}$. Taking

$$
\alpha_{1} \doteq \varphi_{1} \circ \alpha \circ\left(\mathrm{id}+\varphi_{2} \circ \alpha\right)^{-1}, \quad \text { and } \quad \alpha_{2} \doteq \varphi_{2} \circ \alpha
$$

we immediately have

$$
\begin{aligned}
\left(\alpha_{1}+\mathrm{id}\right) \circ\left(\alpha_{2}+\mathrm{id}\right) & =\alpha_{1} \circ\left(\alpha_{2}+\mathrm{id}\right)+\alpha_{2}+\mathrm{id} \\
& =\varphi_{1} \circ \alpha \circ\left(\mathrm{id}+\varphi_{2} \circ \alpha\right)^{-1} \circ\left(\mathrm{id}+\varphi_{2} \circ \alpha\right)+\varphi_{2} \circ \alpha+\mathrm{id} \\
& =\varphi_{1} \circ \alpha+\varphi_{2} \circ \alpha+\mathrm{id}=\alpha+\mathrm{id} .
\end{aligned}
$$

We observe that when $\alpha \in \mathcal{K}_{\infty}$, we have $\alpha_{1}, \alpha_{2} \in \mathcal{K}_{\infty}$.

\section{A.8 Proof of Lemma 25}

Since $\alpha-\mathrm{id} \in \mathcal{K}_{\infty}$, we know that the interval [1, $\left.\alpha(1)\right)$ is nonempty. Let the function $\bar{\alpha}:[1, \alpha(1)) \rightarrow[1, \lambda)$ be continuous, strictly increasing, and satisfy the endpoint conditions

$$
\bar{\alpha}(1)=1 \text { and } \lim _{s \nearrow \alpha(1)} \bar{\alpha}(s)=\lambda
$$

Since $\alpha \in \mathcal{K}_{\infty}$, by a slight abuse of notation, we observe that the interval mappings satisfy $\alpha([1, \alpha(1))) \mapsto\left[\alpha(1), \alpha^{2}(1)\right)$ and $\alpha^{-1}([1, \alpha(1))) \mapsto\left[\alpha^{-1}(1), 1\right)$ and, in general

$$
\alpha^{k}([1, \alpha(1))) \mapsto\left[\alpha^{k}(1), \alpha^{k+1}(1)\right)
$$

It is clear that the intervals $\left[\alpha^{k}(1), \alpha^{k+1}(1)\right), k \in \mathbb{Z}$, are disjoint and cover $\mathbb{R}_{>0}$. Since $\alpha$ is monotonically increasing, it follows that, for $s>0$, there are unique values $(k(s), r(s)) \in \mathbb{Z} \times[1, \alpha(1))$ such that

$$
s=\alpha^{k(s)}(r(s))
$$

We define $\hat{\alpha}: \mathbb{R}_{\geq 0} \rightarrow \mathbb{R}_{\geq 0}$ as

$$
\hat{\alpha}(s) \doteq \begin{cases}\lambda^{k(s)} \bar{\alpha}(r(s)), & s>0 \\ 0, & s=0 .\end{cases}
$$


We first verify that $\hat{\alpha} \in \mathcal{K}_{\infty}$. Continuity of $\hat{\alpha}$ on the intervals $\left[\alpha^{k}(1), \alpha^{k+1}(1)\right)$ follows from the assumption of continuity of $\bar{\alpha}$, while continuity at the endpoints of the intervals follows from (54). Furthermore, we see that $\hat{\alpha}$ is continuous at the origin since $\lambda>1$ and $k(s) \rightarrow-\infty$ as $s \rightarrow 0$. The fact that $\hat{\alpha}$ is strictly increasing follows from the assumption that $\bar{\alpha}$ is strictly increasing. That $\hat{\alpha}$ is unbounded follows from the facts that $\lambda>1, k(s) \rightarrow \infty$ as $s \rightarrow \infty$, and $\bar{\alpha}(r) \geq 1$ for $r \in[1, \alpha(1))$.

We now verify (16). From (55) we see that for all $s \in \mathbb{R}_{\geq 0}$

$$
\alpha(s)=\alpha \circ \alpha^{k(s)}(r(s))=\alpha^{k(s)+1}(r(s)) .
$$

Consequently, with the definition (56)

$$
\begin{aligned}
\hat{\alpha} \circ \alpha(s) & =\hat{\alpha} \circ \alpha^{k(s)+1}(r(s))=\lambda^{k(s)+1} \bar{\alpha}(r(s)) \\
& =\lambda\left(\lambda^{k(s)} \bar{\alpha}(r(s))\right)=\lambda \hat{\alpha}(s) .
\end{aligned}
$$

\section{A.9 Proof of Corollary 1}

Define $\varphi \doteq(\mathrm{id}-\alpha)^{-1}$ and note that $\varphi \in \mathcal{K}_{\infty}$. Manipulating the definition of $\varphi$, we obtain $\varphi(s)-s=\alpha \circ \varphi(s)$ for all $s \in \mathbb{R}_{\geq 0}$. Since $\varphi, \alpha \in \mathcal{K}_{\infty}$, we have that $(\varphi-\mathrm{id}) \in \mathcal{K}_{\infty}$. We then appeal to Lemma 25 with $\lambda^{-1}>1$ and $\varphi \in \mathcal{K}_{\infty}$ to obtain $\hat{\alpha} \in \mathcal{K}_{\infty}$ such that

$$
\hat{\alpha} \circ \varphi(r)=\lambda^{-1} \hat{\alpha}(r), \quad \forall r \in \mathbb{R}_{\geq 0}
$$

Substituting the definition of $\varphi$ yields

$$
\lambda \hat{\alpha} \circ(\mathrm{id}-\alpha)^{-1}(r)=\hat{\alpha}(r), \quad \forall r \in \mathbb{R}_{\geq 0} .
$$

Taking $s=(\mathrm{id}-\alpha)(r)$ then yields (17).

\section{A.10 Proof of Lemma 27}

The proof in one direction follows that in [38]. The converse uses a similar argument but illustrates the care that must be taken when using class- $\mathcal{K}$ functions rather than class- $\mathcal{K}_{\infty}$ functions.

To obtain a contradiction, suppose there exists $s^{*} \in\left[0, \alpha_{2}(c)\right]$ such that

$$
\alpha_{2} \circ \alpha_{1}\left(s^{*}\right)>s^{*} \text {. }
$$

Then, since $\alpha_{2} \in \mathcal{K}$ is invertible on its range, $\alpha_{2}^{-1}\left(s^{*}\right)$ is well defined and therefore $\alpha_{1}\left(s^{*}\right)>\alpha_{2}^{-1}\left(s^{*}\right) \doteq r^{*} \in[0, c]$. Therefore,

$$
\alpha_{1} \circ \alpha_{2}\left(r^{*}\right)=\alpha_{1}\left(s^{*}\right)>\alpha_{2}^{-1}\left(s^{*}\right)=r^{*}
$$

which contradicts $\alpha_{1} \circ \alpha_{2}(s) \leq s$ for all $s \in[0, c]$. 
To show the converse implication, we again argue by contradiction. Suppose there exists $s^{*} \in[0, c]$ such that

$$
\alpha_{1} \circ \alpha_{2}\left(s^{*}\right)>s^{*}
$$

Define $a_{1}=\lim _{s \rightarrow \infty} \alpha_{1}(s)$. We consider three cases.

Case 1: Suppose $a_{1} \geq c$. Then $r^{*} \doteq \alpha_{1}^{-1}\left(s^{*}\right)$ is well defined. We see that (57) implies $\alpha_{2}\left(s^{*}\right)>\alpha_{1}^{-1}\left(s^{*}\right)=r^{*}$ so that $r^{*} \in\left[0, \alpha_{2}(c)\right]$. Therefore, (57) implies

$$
\alpha_{2} \circ \alpha\left(r^{*}\right)=\alpha_{2}\left(s^{*}\right) \leq \alpha_{1}^{-1}\left(s^{*}\right)=r^{*}
$$

which contradicts $\alpha_{2} \circ \alpha_{1}(s) \leq s$ for all $s \in[0, \alpha(c)]$.

Case 2: Suppose $a_{1}<c$ and $s^{*} \in\left[0, a_{1}\right)$ then, as in Case $1, r^{*}=\alpha_{1}^{-1}\left(s^{*}\right)$ is well defined and a contradiction follows by the same argument.

Case 3: Finally, suppose $a_{1}<c$ and $s^{*} \in\left[a_{1}, c\right]$. Then

$$
\alpha_{1} \circ \alpha_{2}\left(s^{*}\right)<a_{1} \leq s^{*}
$$

which immediately contradicts (57) and thus proves the lemma.

We note that the same proof can be used to demonstrate Corollary 2 without needing to account for the values for which $\alpha_{2}^{-1}$ is well defined.

\section{A.11 Proof of Lemma 31}

The proof considers two cases: $c=\infty$ and $c<\infty$.

Consider $c=\infty$, so that (24) holds, and define $a \doteq \lim _{s \rightarrow \infty} \alpha(s)$. We see that $\alpha \circ \gamma(s) \leq s$ and (25) imply

$$
\alpha^{\ominus} \circ \alpha \circ \gamma(s)=\gamma(s) \leq \alpha^{\ominus}(s), \quad \forall s \in \overline{\mathbb{R}}_{\geq 0} .
$$

Let $r \in[0, a]$ be given by $r=\alpha(s)$. Then (25) and (58) yield

$$
\gamma \circ \alpha(s)=\gamma(r) \leq \alpha^{\ominus}(r)=\alpha^{\ominus} \circ \alpha(s)=s .
$$

To demonstrate the converse, we first note that since for all $s \in \overline{\mathbb{R}}_{\geq 0}$ we have $\alpha(s) \leq a$, then for $s \geq a$ we have $s \geq a \geq \alpha \circ \gamma(s)$. Hence, we restrict attention to $s \in[0, a)$. Let $\alpha(r)=s$ so that $r=\alpha^{\ominus}(s)$. Then $\gamma \circ \alpha(r) \leq r$ implies $\gamma(s) \leq \alpha^{\ominus}(s)$. Applying $\alpha$ to both sides of this inequality and using (25) we obtain

$$
\alpha \circ \gamma(s) \leq \alpha \circ \alpha^{\ominus}(s) \leq s, \quad \forall s \in[0, a)
$$

which completes the proof for $c=\infty$. 
Now consider $c<\infty$. The proof for both implications proceeds by contradiction. To obtain a contradiction in the first instance, suppose there exists $s^{*} \in \overline{\mathbb{R}}_{\geq 0}$ so that $\gamma \circ \alpha\left(s^{*}\right)>s^{*}$. First consider $s^{*}<c$. Then there exists $r^{*}<\infty$ so that $\alpha\left(s^{*}\right)=r^{*}$. This then implies

$$
\gamma\left(r^{*}\right)=\gamma \circ \alpha\left(s^{*}\right)>s^{*}=\alpha^{-1}\left(r^{*}\right)
$$

and therefore $\alpha \circ \gamma\left(r^{*}\right)>r^{*}$, which is a contradiction.

Next, consider $s^{*} \geq c$. In this case, $\alpha\left(s^{*}\right)=\infty$ so that

$$
\gamma \circ \alpha\left(s^{*}\right)=\gamma(\infty)>s^{*} \geq c,
$$

which contradicts the condition that $\gamma(\infty)<c$. This then proves the first implication.

To prove the converse, suppose there exists $s^{*} \in \overline{\mathbb{R}}_{\geq 0}$ so that $\alpha \circ \gamma\left(s^{*}\right)>s^{*}$. Since $\gamma(\infty)<c$, we see that

$$
\infty>\alpha \circ \gamma\left(s^{*}\right)>s^{*} \text {. }
$$

For $c<\infty$ the domain of $\alpha^{-1}$ is $[0, \infty)$ and so we have

$$
\alpha^{-1} \circ \alpha \circ \gamma\left(s^{*}\right)=\gamma\left(s^{*}\right)>\alpha^{-1}\left(s^{*}\right) \text {. }
$$

Observe that $c<\infty$ implies $\lim _{s \rightarrow c} \alpha(s)=\infty$ and so there exists $r^{*} \in \overline{\mathbb{R}}_{\geq 0}$ so that $\alpha\left(r^{*}\right)=s^{*}$. Consequently,

$$
\gamma \circ \alpha\left(r^{*}\right)>r^{*}
$$

which provides our final contradiction and hence completes the proof.

\section{References}

1. Albertini F, Sontag ED (1999) Continuous control-Lyapunov functions for asymptotically controllable time-varying systems. Int J Control 72:1630-1641

2. Angeli D, Ingalls B, Sontag ED, Wang Y (2004) Separation principles for input-output and integralinput-to-state stability. SIAM J Control Optim 43(1):256-276

3. Angeli D, Sontag ED, Wang Y (2000) A characterization of integral input-to-state stability. IEEE Trans Autom Control 45(6):1082-1097

4. Barbashin EA, Krasovskii NN (1952) On the stability of motion in the large. Dokl Acad Nauk 86:453456

5. Clarke FH, Ledyaev YS, Stern RJ (1998) Asymptotic stability and smooth Lyapunov functions. J Differ Equ 149:69-114

6. Dashkovskiy SN, Rüffer BS, Wirth FR (2008) Applications of the general Lyapunov ISS small-gain theorem for networks. In: Proceedings of the 47th IEEE Conference on Decision and Control, Cancun, Mexico, pp 25-30

7. Dower PM, Kellett CM (2008) A dynamic programming approach to the approximation of nonlinear $\mathcal{L}_{2}$-gain. In: Proceedings of the 47th IEEE Conference on Decision and Control, Cancun, Mexico, pp $1-6$

8. Geiselhart R, Wirth F (2013) On maximal gains guaranteeing a small-gain condition (Submitted) 
9. Grüne L (2002) Asymptotic behavior of dynamical systems under perturbation and discretization. Lecture notes in mathematics. Springer, Berlin

10. Grüne L (2002) Input-to-state dynamical stability and its Lyapunov function characterization. IEEE Trans Autom Control 47(9):1499-1504

11. Hahn W (1959) Theorie und Anwendung der direkten Methode von Ljapunov. Ergebnisse der Mathematik und ihrer Grenzgebiete. Springer, 1963. English translation: Theory and application of Liapunov's Direct Method, Prentice-Hall, Englewood Cliffs

12. Hahn W (1967) Stability of motion. Springer, Berlin

13. Hardy G, Littlewood JE, Polya G (1989) Inequalities, 2nd edn. Cambridge University Press, Cambridge

14. Ito H (2012) Necessary conditions for global asymptotic stability of networks of iISS systems. Math Control Signals Syst 24:55-74

15. Ito H, Jiang Z-P, Dashkovskiy SN, Rüffer BS (2013) Robust stability of networks of iISS systems: construction of sum-type Lyapunov functions. IEEE Trans Autom Control 58(5):1192-1207

16. Jiang Z-P, Mareels IMY (1997) A small-gain control method for nonlinear cascaded systems with dynamic uncertainties. IEEE Trans Autom Control 42(3):292-308

17. Jiang Z-P, Teel AR, Praly L (1994) Small-gain theorem for ISS systems and applications. Math Control Signals Syst 7(2):95-120

18. Kellett CM, Teel AR (2004) Discrete-time asymptotic controllability implies smooth control-Lyapunov function. Syst Control Lett 52(5):349-359

19. Kellett CM, Teel AR (2004) Weak converse Lyapunov theorems and control Lyapunov functions. SIAM J Control Optim 42(6):1934-1959

20. Krstić M, Li Z-H (1998) Inverse optimal design of input-to-state stabilizing nonlinear controllers. IEEE Trans Autom Control 43(3):336-350

21. Lakshmikantham V, Leela S, Martynyuk AA (1989) Stability analysis of nonlinear systems. Marcel Dekker, New York

22. Lin Y, Sontag ED, Wang Y (1996) A smooth converse Lyapunov theorem for robust stability. SIAM J Control Optim 34(1):124-160

23. Logemann H, Sarkans E (2012) Input-to-state stability for discrete-time Lur'e systems. In: Proceedings of Mathematical Theory of Networks and Systems

24. Lyapunov AM (1892) The general problem of the stability of motion. Math Soc Kharkov (Russian). (English Translation. Int J Control 55:531-773 (1992))

25. Massera JL (1949) On Liapounoff's conditions of stability. Ann Math 50:705-721

26. Massera JL (1956) Contributions to stability theory. Ann Math 64:182-206. (Erratum. Annals of Mathematics, 68:202, 1958)

27. Moisseiev N (1937) Über Stabilitätswahrscheinlichkeitsrechnung. Mathematische Zeitschrift 42:513537

28. Müller M (1926) Über das Fundamentaltheorem in der Theorie der gewöhnlichen Differentialgleichungen. Mathematische Zeitschrift 26:619-645

29. Praly L, Jiang Z-P (1993) Stabilization by output feedback for systems with ISS inverse dynamics. Syst Control Lett 21(1):19-34

30. Praly L, Wang Y (1996) Stabilization in spite of matched unmodeled dynamics and an equivalent definition of input-to-state stability. Math Control Signals Syst 9:1-33

31. Riesz F, Nagy B Sz (1955) Functional analysis. Frederick Ungar Publishing Co., New York, Republished by Dover Publications Inc, 1990

32. Rouche N, Habets P, Laloy M (1977) Stability theory by Liapunov's direct method. Springer, Berlin

33. Rüffer BS (2007) Monotone dynamical systems, graphs, and stability of large-scale interconnected systems. PhD thesis, Universität Bremen

34. Sánchez DA (1968) Ordinary differential equations and stability theory. W. H,Freeman and Company, San Francisco

35. Sontag ED (1989) Smooth stabilization implies coprime factorization. IEEE Trans Autom Control 34(4):435-443

36. Sontag ED (1998) Comments on integral variants of ISS. Syst Control Lett 34(1-2):93-100

37. Sontag ED, Wang Y (2000) Lyapunov characterizations of input to output stability. SIAM J Control Optim 39(1):226-249

38. Teel A, Praly L (1995) Tools for semiglobal stabilization by partial state and output feedback. SIAM J Control Optim 33(5):1443-1488 
39. Teel AR (1996) A nonlinear small gain theorem for the analysis of control systems with saturation. IEEE Trans Autom Control 41(9):1256-1270

40. Teel AR (1998) Connections between Razumikhin-type theorems and the ISS nonlinear small gain theorem. IEEE Trans Autom Control 43(7):960-964

41. Vidyasagar M (1978) Nonlinear systems analysis. Prentice-Hall, Englewood Cliffs

42. Vidyasagar M (1993) Nonlinear systems analysis, 2nd edn. Prentice-Hall, Englewood Cliffs 